ENSLAPP-A-434/93

TUM-TH-160/93

NTUA 43/93

hep-th/9403131

March 1994

\title{
Quantum Cosmology with Yang-Mills Fields
}

\author{
D. Kapetanakis ${ }^{1}$, G. Koutsoumbas ${ }^{2}$, A. Lukas ${ }^{3}$ and P. Mayr ${ }^{3}$ \\ ${ }^{1}$ Laboratoire de Physique Théorique \\ Chemin de Bellevue, F-74941 \\ Annecy-le-Vieux Cedex, France \\ ${ }^{2}$ Physics Department \\ National Technical University \\ 15780 Zografou, Athens, Greece \\ ${ }^{3}$ Physik Department \\ Technische Universität München \\ D-85747 Garching, Germany
}

\begin{abstract}
We examine an extension of the ideas of quantum cosmology and, in particular, the proposal of Hartle and Hawking for the boundary conditions of the Universe, to models which incorporate Yang-Mills fields. Inhomogeneous perturbations about a homogeneous, isotropic minisuperspace background model are considered, by expanding the Yang-Mills fields in harmonics of the spatial directions which are taken to be three-spheres. The expansions are made explicit for $S O(N)$ gauge fields thereby obtaining formulae compatible with the formalism conventionally used in quantum cosmology. We apply these results to the gauge group $S O(3)$ and derive the Lagrangian and the semi-classical wave function for this special case.
\end{abstract}




\section{Introduction}

Recently we have witnessed a great activity in the interplay between high energy physics and cosmology. The question of the initial conditions which in the early universe gave origin to the universe that we observe is clearly one of the main questions asking for clarification. Obviously, progress in such a direction needs an understanding of quantum gravity which is still lacking. On the other hand quantum cosmology provides a useful setting to discuss initial conditions and quantum gravity ideas. The most interesting results in this area were obtained from the so-called minisuperspace models where the infinite number of modes of the gravitational and matter fields was reduced by freezing out all the modes except of a finite number which are assumed to dominate. The quantization then proceeds through the application of the ADM [1] formalism, thus obtaining the Wheeler-DeWitt equation as the Hamiltonian constraint and interpreting the conjugate momenta as operators, plus momentum constraints. It is clear that in order to go beyond the minisuperspace analysis one has to include the infinite number of the field modes, treated as frozen previously. This is usually done by treating these modes as perturbations around the minisuperspace degrees of freedom.

In this context, Hartle and Hawking [2, 3] worked out the no-boundary proposal. An alternative suggestion is the so-called tunneling boundary condition 때. Excellent reviews already exist on the subject [5]. However, in most of the minisuperspace models and their extensions including perturbations which exist so far, the matter source is taken to be a scalar field [3]. Also fermion fields [6] and electromagnetism [7] have been considered. Though this list contains fundamental fields, the absence of non-Abelian gauge fields is striking, especially if one considers that the evolution of the very early universe is dominated by gravitational and non-Abelian gauge fields. Recently a minisuperspace model including gauge fields was constructed [8] and the wave function for this model was determined [9]. A key ingredient of this model was the theory of symmetric fields [10, 11], which has been used before in the spontaneous compactification and dimensional reduction of higher dimensional models, to obtain $S O(4)$-symmetric ansätze for the fields in a $R \times S^{3}$ topology. Let us remark here that it has been shown, at least in the case of a scalar field coupled to gravity, that if one considers perturbations of a minisuperspace model with three-spheres as spatial sections, any wave function, the object that we are mainly interested in, has to be $S O(4)$ invariant [12].

The main subject of this paper is precisely to consider the above model beyond minisuperspace, including all the modes of the fields. For that purpose, we use the tool of harmonic analysis of symmetric fields on homogeneous spaces [11, 13], including to lowest non-trivial order the inhomogeneous modes of the gauge fields, while treating the 
homogeneous degrees exactly. The method of harmonic expansion around a symmetric background configuration allows us to develop an analysis for arbitrary gauge group. Furthermore, and more importantly, it allows an analysis of the full realistic system of gauge fields coupled to fermions and scalars in a unified way. Therefore, using the method of symmetric fields one can consider the study of the coupled matter sources as well as supergravity theories coupled to Yang-Mills (YM), something which we consider as a continuation of our work.

The organization of our paper is the following. In section 2 we review the construction of the symmetric model. In section 3 we apply the tools of the harmonic analysis on homogeneous spaces and symmetric fields on our particular system, i.e. a symmetric gauge field on $R \times S^{3}$ with gauge group $G=S O(N)$. Then we specialize on the case $G=S O(3)$. In section 4 we present the perturbed model and in section 5 we consider the equations of the tensor, vector and scalar modes and their contribution to the wave function. We examine a particular case, namely the full system of perturbations, when the classical background field is fixed to its minimum value. Furthermore we discuss the interactions between gauge and gravitational perturbations, which might be quite interesting for the discussion of cosmological models and anisotropies in the cosmological background radiation. Finally in section 7 we present our conclusions. We also provide two appendices with details about the theory of symmetric fields and harmonic analysis on $S^{3}$.

\section{General Formalism and the Symmetric Model}

Let us start by reviewing the relevant parts of the work already existing on the subject; this will allow us to introduce the problem and fix the notation. We will deal with closed universes of the form $M=R \times B$, where $B$ is a compact three-dimensional hypersurface which eventually will be the three-sphere; time $t$ takes values in $R$. For the description of such a universe, a $3+1$ formulation of the four dimensional Einstein theory is appropriate. Such a description, corresponding to an embedding of a three surface in the four dimensional spacetime is given by the $\mathrm{ADM}$ formalism [四] $(\mu, \nu=0, \ldots, 3, \alpha, \beta=1, \ldots, 3)$ :

$$
\begin{aligned}
d s^{2} & =g_{\mu \nu} d x^{\mu} d x^{\nu} \\
& =-\left(N^{2}-N^{\alpha} N_{\alpha}\right) d t^{2}+2 N_{\alpha} d x^{\alpha} d t+h_{\alpha \beta} d x^{\alpha} d x^{\beta} .
\end{aligned}
$$

The quantities $N$ and $N_{\alpha}$ are called lapse and shift functions, respectively, while $h_{\alpha \beta}$ is a three-dimensional metric corresponding to $B$. The action of the Einstein-Yang-Mills 
(EYM) system that we are going to consider has generally the form :

$$
\begin{aligned}
S & =S_{g r}+S_{Y M}, \\
S_{G R} & =\frac{m_{P}^{2}}{16 \pi}\left[\int_{M} d^{4} x(-g)^{\frac{1}{2}}(R-2 \lambda)+2 \int_{\partial M} d^{3} x h^{\frac{1}{2}} K\right], \\
S_{Y M} & =\frac{1}{8 e^{2}} \int_{M} d^{4} x(-g)^{\frac{1}{2}} \operatorname{Tr}\left(F_{\mu \nu} F^{\mu \nu}\right),
\end{aligned}
$$

where $R$ is the scalar curvature corresponding to $g_{\mu \nu}, h \equiv \operatorname{det}\left(h_{\alpha \beta}\right)$ and $K$ is the trace of the extrinsic curvature $K_{\alpha \beta}$ of $B \equiv \partial M$. It may be shown that eq. (3) may be brought to the form

$$
S_{G R}=\frac{m_{P}^{2}}{16 \pi} \int d^{3} x d t N\left[G^{\alpha \beta \gamma \delta} K_{\alpha \beta} K_{\gamma \delta}+\sqrt{h}\left({ }^{3} R-2 \lambda\right)\right],
$$

where ${ }^{3} R$ is the curvature of $B$ and $G^{\alpha \beta \gamma \delta}$ is the DeWitt metric given by

$$
G^{\alpha \beta \gamma \delta}=\frac{1}{2} \sqrt{h}\left(h^{\alpha \gamma} h^{\beta \delta}+h^{\alpha \delta} h^{\beta \gamma}-2 h^{\alpha \beta} h^{\gamma \delta}\right) .
$$

The corresponding $3+1$ - decomposition of eq. (画) is

$$
\begin{aligned}
S_{Y M}= & \frac{1}{8 e^{2}} \int d^{3} x d t \sqrt{h} N \operatorname{tr}\left[\left(h^{\alpha \gamma} h^{\beta \delta}-2 h^{\alpha \gamma} h^{\beta \epsilon} h^{\delta \rho} \frac{N_{\epsilon} N_{\rho}}{N^{2}}\right) F_{\alpha \beta} F_{\gamma \delta}\right. \\
& \left.-2 \frac{h^{\alpha \beta}}{N^{2}} F_{0 \alpha} F_{0 \beta}-4 h^{\alpha \beta} h^{\gamma \delta} \frac{N_{\delta}}{N^{2}} F_{\alpha 0} F_{\beta \gamma}\right] .
\end{aligned}
$$

One may proceed as usual to determine the conjugate momenta and the Hamiltonian. It turns out that the Hamiltonian is a sum of constraints :

$$
H=\int_{\partial M} d^{3} x \sqrt{h}\left(N H_{0}+N^{\alpha} H_{\alpha}+\operatorname{tr}\left(A_{0} H_{Y M}\right)\right) .
$$

The lapse and shift functions play the role of Lagrange multipliers, enforcing the constraints which correspond to Einstein's equations, namely the momentum constraint $H_{\alpha}$ and the Hamiltonian constraint $H_{0}$. Gauge invariance is guaranteed by the constraint $H_{Y M}$ with the time component $A_{0}$ of the gauge field as the corresponding Lagrange multiplier.

Now we are ready to consider the quantized model, which will be described by a wave functional $\Psi\left[h_{\alpha \beta}(x), A(x)\right]$ of the metric $h_{\alpha \beta}(x)$ and the gauge field $A(x)$. The constraints $H_{0}, H_{\alpha}$ and $H_{Y M}$ are expressed by demanding that their operator versions annihilate the wave functional $\Psi\left[h_{\alpha \beta}(x), A(x)\right]$. They ensure the invariance of the wave functional under reparametrizations and gauge transformations. The solution of the Wheeler-DeWitt equation $H_{0} \Psi=0$ subject to the other constraints lies at the heart of the subject of quantum cosmology. 
We proceed by reducing the infinite dimensional superspace $\left(h_{\alpha \beta}(x), A(x)\right)$ to a finite dimensional set, the so called minisuperspace. Some of the very common defining conditions of minisuperspace are :

1. The lapse is taken to be homogeneous, i.e. $N=N(t)$.

2. The shift $N_{\alpha}$ is set to zero.

3. The three-metric $h_{\alpha \beta}$ is constrained to be homogeneous and isotropic.

In quantum cosmology one is usually interested in closed universes. This, together with the above requirements fixes the spatial sections of $M$ to be $S^{3}$. Another way to single out this case is to impose $S O(4) \equiv S U(2) \times S U(2)$ invariance on the metric of $B \equiv \partial M$ which leads to円

$$
g_{00}=-\sigma^{2} N(t)^{2}, \quad g_{a b} \equiv h_{a b}=\sigma^{2} a(t)^{2} \delta_{a b}, \quad g_{0 a}=0 .
$$

An additional factor $\sigma^{2} \equiv 8 \pi / m_{P}^{2}$ has been introduced for convenience. Using this metric we obtain

$$
S_{G R_{0}}=-3 V_{S^{3}} \int d t\left[\frac{a}{N} \dot{a}^{2}-N a+H^{2} N a^{3}\right],
$$

where $H^{2} \equiv 8 \pi \lambda / 3 m_{P}^{2}$ and $V_{S^{3}}=2 \pi^{2}$ is the volume of the three sphere with radius one.

For the matter sector, the following requirement is imposed, taking advantage of the gauge freedom : the change of the Yang-Mills field for motion on $B$ at fixed $t$ is allowed to be a gauge transformation rather than zero. In other words, we are imposing $S O(4) \equiv$ $S U(2) \times S U(2)$ invariance on the gauge field modulo gauge transformation [11]. For the gauge group $G=S O(N)$, according to the rules given in appendix A, the gauge potential is

$$
A(t) \equiv A_{0}(t) d t+A_{a}(t) \omega^{a}
$$

with

$$
\begin{gathered}
A_{0}(t)=\frac{1}{2} \Lambda^{I J}(t) T_{I J}, \\
A_{a}(t)=\left[1+\chi_{0}(t)\right] T_{a}+\chi^{I}(t) T_{a I},
\end{gathered}
$$

and the Maurer-Cartan forms $\omega^{a}$ on the coset $S^{3} \cong S U(2)$ satisfying $d \omega^{a}+\epsilon_{b c}^{a} \omega^{b} \wedge \omega^{c}=$ 0 . The matrices $T_{A B}$ are representation matrices of $S O(N)$ in correspondence with the decomposition $S O(N) \supset S O(3) \times S O(N-3)$

$$
\frac{N(N-1)}{2}=\left(\begin{array}{c}
(3,1) \\
T_{a}
\end{array}+\left(1, \frac{(N-3)(N-4)}{2}\right)+(3, N-3),\right.
$$

${ }^{1}$ We will use Latin letters from the beginning of the alphabet to denote representation indices taking values in the orthonormal bundle. 
which satisfy the following non-trivial commutation relations :

$$
\begin{aligned}
{\left[T_{a}, T_{b}\right] } & =\epsilon_{a b c} T_{c}, \\
{\left[T_{a}, T_{b K}\right] } & =\epsilon_{a b c} T_{c K}, \\
{\left[T_{I J}, T_{K L}\right] } & =-\delta_{I K} T_{J L}+\delta_{I L} T_{J K}+\delta_{J K} T_{I L}-\delta_{J L} T_{I K}, \\
{\left[T_{I J}, T_{a K}\right] } & =\delta_{J K} T_{a I}-\delta_{I K} T_{a J} .
\end{aligned}
$$

The functions $\chi_{0}(t), \chi_{I}(t)$ and $\Lambda^{k m}(t)$ are arbitrary. We should also note that the isomorphic image of the isotropy group $S O(3)$ in the gauge group $S O(N)$ is taken to be the group generated by the three antisymmetric matrices $T_{i}$. We see in this example how the $S O(4)$ symmetry requirement for the gauge fields has reduced an infinite number of degrees of freedom down to a fairly small number of functions. Moreover, for $S O(4)$ symmetric fields the Lagrangian does not depend on the spatial coordinates, so the integration over the three sphere will just yield the volume $V_{S^{3}}=2 \pi^{2}$ of $S^{3}$ as an overall factor. Taking this into account the action (17), after substituting

$$
\begin{aligned}
& F_{0 a}=\dot{\chi}_{0} T_{a}+2 \dot{\chi}^{I} T_{a I}-2 \chi_{I} \Lambda^{I J} T_{a J}, \\
& F_{a b}=\left(1-\chi_{0}^{2}-\chi^{2}\right) \epsilon_{a c b} T_{c}-2 \chi_{0} \epsilon_{a c b} \chi^{I} T_{c I}
\end{aligned}
$$

for the field strength becomes

$$
S_{Y M_{0}}=V_{S^{3}} \frac{3}{2 e^{2}} \int d t\left[\frac{a}{N}\left(\left(\frac{d \chi_{0}}{d t}\right)^{2}+\left(\mathcal{D}_{t} \boldsymbol{\chi}\right)^{2}\right)+2 \frac{N}{a} V\left(\chi_{0}, \chi\right)\right],
$$

where

$$
\begin{gathered}
\mathcal{D}_{t} \chi \equiv \frac{d}{d t} \chi+\Lambda \chi \\
V\left(\chi_{0}, \chi\right) \equiv \frac{1}{2}\left(\chi_{0}^{2}+\chi^{2}-1\right)^{2}+2 \chi_{0}^{2} \chi^{2} .
\end{gathered}
$$

Given the action $S_{0}=S_{G R_{0}}+S_{Y M_{0}}$, we may find the conjugate momenta and the Hamiltonian. It turns out that (dropping the factor $3 V_{S^{3}} / e^{2}$ for simplicity)

$$
\pi_{a}=-\frac{a}{N} \dot{a}, \quad \pi_{\chi_{0}}=\frac{a}{N} \dot{\chi_{0}}, \quad \pi \chi=\frac{a}{N} \mathcal{D}_{t} \chi .
$$

The Hamiltonian reads

$$
H \equiv \frac{1}{2} \frac{N}{a}\left[-\pi_{a}^{2}-a^{2}+H^{2} a^{4}+\pi_{\chi_{0}}^{2}+\pi_{\chi}^{2}+2 V\left(\chi_{0}, \chi\right)\right],
$$

giving rise to the Wheeler-DeWitt equation

$$
\frac{1}{2}\left[a^{-p} \frac{\partial}{\partial a}\left(a^{p} \frac{\partial}{\partial a}\right)-a^{2}+H^{2} a^{4}-\frac{\partial^{2}}{\partial \chi_{0}^{2}}-\frac{\partial^{2}}{\partial \chi^{2}}+2 V\left(\chi_{0}, \boldsymbol{\chi}\right)\right] \psi\left(a, \chi_{0}, \boldsymbol{\chi}\right)=E \psi\left(a, \chi_{0}, \boldsymbol{\chi}\right) .
$$


The parameter $p$ enters to take care of the operator ordering problem [2]. For the case under investigation the gauge constraints $H_{Y M}$ are related to the Lagrange multipliers $\Lambda$ (cf. eq. (8)). They boil down to the classical condition

$$
\chi_{I} \pi_{\chi_{J}}-\chi_{J} \pi_{\chi_{I}}=0
$$

or, in the quantum language

$$
\left(\chi_{I} \frac{\partial}{\partial \chi_{J}}-\chi_{J} \frac{\partial}{\partial \chi_{I}}\right) \psi\left(a, \chi_{0}, \boldsymbol{\chi}\right)=0 .
$$

Based on the fact that the gravity and the gauge parts of the problem decouple, the classical behavior has been determined for a general $S O(N)$ gauge group. It exhibits interesting features, for example wormhole solutions arise upon consideration of a Euclideanized version. Moreover, in the special case $N=3$, the wave function has been determined in the semi-classical approximation by Bertolami and Mourão [9]. Writing the separation ansatz

$$
\Psi\left(a, \chi_{0}\right)=\sum_{n} C_{n}(a) U_{n}\left(\chi_{0}\right),
$$

where $C_{n}(a)$ is the gravitational wave-function obtained by Hartle and Hawking [3], $U_{n}\left(\chi_{0}\right)$ is given by

$$
\begin{aligned}
& U\left(\chi_{0}\right)=A \exp \left(-\frac{V_{S 3}}{e^{2}}\left(3\left(\chi_{0}+1\right)^{2}-\left(\chi_{0}+1\right)^{3}\right)\right), \text { for } \quad \chi_{0}<-1, \\
& U\left(\chi_{0}\right)=B \exp \left(-\frac{V_{S 3}}{e^{2}}\left(3\left(\chi_{0}+1\right)^{2}-\left(\chi_{0}+1\right)^{3}\right)\right) \\
& +B \exp \left(-\frac{V_{S 3}}{e^{2}}\left(3\left(\chi_{0}-1\right)^{2}+\left(\chi_{0}-1\right)^{3}\right)\right), \quad \text { for }\left|\chi_{0}\right| \leq 1 \text {, } \\
& U\left(\chi_{0}\right)=A \exp \left(-\frac{V_{S 3}}{e^{2}}\left(3\left(\chi_{0}-1\right)^{2}+\left(\chi_{0}-1\right)^{3}\right)\right), \text { for } \quad \chi_{0}>1 .
\end{aligned}
$$

Here $A=(1+\exp (-4 / 3)) B$ and $B$ is a normalization factor. The Hartle-Hawking boundary proposal has been imposed to arrive at these solutions by requiring that $\chi_{0}(\eta=$ $-\infty)=\mp 1$. Furthermore $\chi_{0}(\eta=0)=\chi_{0}$ is the value at the given three-surface and $\eta$ is the conformal time.

\section{Harmonic Analysis}

A very interesting issue is to see what happens beyond the minisuperspace approximation. This has been done by Halliwell and Hawking [3] for the case of a scalar field coupled to gravity. They consider perturbations around the minisuperspace model, considering the following forms for the various fields involved

$$
h_{\alpha \beta}=a^{2}\left(\Omega_{\alpha \beta}+\varepsilon_{\alpha \beta}\right), \quad \phi(y, t)=\phi(t)+\delta \phi(y, t), \quad N(y, t)=N_{0}(t)+\delta N(y, t),
$$


where $\Omega_{\alpha \beta}$ is the metric of the three sphere, allowing also $N_{\alpha}(y, t)$ to take (small) non-zero values 2 . The background quantities $a, \phi, N_{0}$ are treated to all orders, and the perturbations up to second order. The method to handle the perturbations is the use of harmonic expansion. In this paper we would like to examine the problem of perturbations around minisuperspace for the case of the $S O(4)$ symmetric gauge fields referred to above. We therefore split the gauge field

$$
A=A^{(0)}+\bar{A}
$$

into a symmetric part $A^{(0)}$ as it was presented for the group $G=S O(N)$ in the last section, and a perturbation $\bar{A}$. On the other hand, following appendix A, $A_{0}$ (which behaves as a scalar as far as the $S O(4)$ rotations are concerned) and $A_{a}$ can be written in the form

$$
\begin{aligned}
& A_{0}(t, y)=\sum_{m, p q} D_{p q}^{(m)}(L(y)) a_{0} \underset{p q}{(m)}(t) \\
& A_{a}(t, y)=A_{a}^{B}+\sum_{m, p q} D_{p q}^{(m)}(L(y)) \chi_{a}{ }_{p q}^{(m)}(t) .
\end{aligned}
$$

We note that the factors $\sqrt{\frac{d_{m}}{d_{\mathcal{D}}}}$ appearing in equations (A.6) and (A.7) have been absorbed in the coefficients $a_{0} \underset{p q}{(m)}(t)$ and $\chi_{a} \underset{p q}{(m)}(t)$. In these equations the field $A^{(0)}$ can be identified with the background $A^{B}$ plus the $y$-independent parts of the expansions corresponding to the trivial representation $(m=0)$ of $S O(4)$. In order to specify the harmonic analysis of the gauge field we must first solve the constraints given in appendix A for the $D_{p q}^{(m)}$ $S O(4)$ representation matrices.

We decompose the adjoint of the gauge group $G$ under $R=S O(3) \approx S U(2)$ according to the embedding :

$$
\begin{array}{ll}
G & \supset R \times H, \\
\operatorname{adj} G=(3,1)+(1, \operatorname{adj} H)+\sum_{k}\left(R_{k}, H_{k}\right) .
\end{array}
$$

Let us next introduce the generators $T_{a}, T_{L}$ and $T_{r_{k} h_{k}}$ of $G$ in correspondence with the decomposition (32). We write their nontrivial commutation relations as

$$
\begin{aligned}
{\left[T_{a}, T_{b}\right] } & =\epsilon_{a b c} T_{c} \\
{\left[T_{a}, T_{r_{k} h_{k}}\right] } & =D_{r_{k}^{\prime} r_{k}}^{\left(R_{k}\right)}\left(T_{a}\right) T_{r_{k}^{\prime} h_{k}} .
\end{aligned}
$$

The coefficients $a_{0}{ }_{p q}^{(m)}(t)$ and $\chi_{a}{ }_{p q}^{(m)}(t)$ take values in the Lie algebra of $G$ and may be expanded in the basis of the matrices $T_{a}, T_{L}$ and $T_{r_{k} h_{k}}$ defined above. The relevant

\footnotetext{
${ }^{2}$ Here and in the following $y$ denotes the coordinates of the three sphere.
} 
expansions read :

$$
\begin{aligned}
& a_{0}{ }_{p q}^{(m)}(t)=\sum_{c} \alpha_{p q \mid c}^{(m)}(t) T_{c}+\sum_{L} \alpha_{p q \mid L}^{(m)}(t) T_{L}+\sum_{k, r_{k}, h_{k}} \alpha_{p q \mid r_{k} h_{k}}^{(m)\left(R_{k}, H_{k}\right)}(t) T_{r_{k} h_{k}}, \\
& \chi_{a}{ }_{p q}^{(m)}(t)=\sum_{c} \chi_{a, p q \mid c}^{(m)}(t) T_{c}+\sum_{L} \chi_{a, p q \mid L}^{(m)}(t) T_{L}+\sum_{k, r_{k}, h_{k}} \chi_{a, p q \mid r_{k} h_{k}}^{(m)\left(R_{k}, H_{k}\right)}(t) T_{r_{k} h_{k}} .
\end{aligned}
$$

In (36) the indices $r_{k}, h_{k}$ run over the representations $R_{k}, H_{k}$ respectively.

The above expansion has been made with an eye to the solution of the constraints (A.8) and (A.9) in appendix A. Indeed, the constraints can be expressed in terms of the components of $a_{0} \underset{p q}{(m)}(t)$ and $\chi_{a} \underset{p q}{(m)}(t)$ as follows (summation over repeated indices is implied) :

$$
\begin{array}{ll}
D_{p s}^{(m)}\left(T_{c}\right) \alpha_{p q \mid a}^{(m)}(t)-\varepsilon_{c b a} \alpha_{s q \mid b}^{(m)}(t) & =0, \\
D_{p s}^{(m)}\left(T_{c}\right) \alpha_{p q \mid J}^{(m)}(t) & =0, \\
D_{p s}^{(m)}\left(T_{c}\right) \alpha_{p q \mid r_{k} h_{k}}^{(m)\left(R_{k}, H_{k}\right)}(t)-D_{r_{k}^{\prime} r_{k}}^{\left(R_{k}\right)}\left(T_{c}\right) \alpha_{\left.s q \mid r_{k}^{\prime} h_{k}, H_{k}\right)}^{(m)\left(R_{k}\right)}(t) & =0, \\
D_{p s}^{(m)}\left(T_{c}\right) \chi_{a, p q \mid d}^{(m)}(t)+\varepsilon_{c a b} \chi_{b, s q \mid d}^{(m)}(t)+\varepsilon_{c d b} \chi_{a, s q \mid b}^{(m)}(t) & =0, \\
D_{p s}^{(m)}\left(T_{c}\right) \chi_{a, p q \mid J}^{(m)}(t)+\varepsilon_{c a b} \chi_{b, s q \mid J}^{(m)}(t) & =0, \\
D_{p s}^{(m)}\left(T_{c}\right) \chi_{a, p q \mid r_{k} h_{k}}^{(m)\left(R_{k}, H_{k}\right)}(t)+\varepsilon_{c a b} \chi_{b, s q \mid r_{k} h_{k}}^{(m)\left(R_{k}, H_{k}\right)}(t) & \\
-D_{r_{k}^{\prime} h_{k}}^{\left(R_{k}\right)}\left(T_{c}\right) \chi_{a, s q \mid r_{k}^{\prime} h_{k}}^{(m)\left(R_{k}, H_{k}\right)}(t) & =0 .
\end{array}
$$

In this form it is easy to invoke Schur's lemma and find the solution of the constraints. According to the discussion in appendix B, we denote the representation $(m)$ by $\left(j_{L}, j_{R} \mid J\right)$; in this notation the nontrivial solution is written as

$$
\begin{aligned}
& \alpha_{s q \mid c}^{\left(j_{L}, j_{R} \mid 1\right)}=\delta_{s c} \alpha_{q}^{\left(j_{L}, j_{R} \mid 1\right)}, \\
& \alpha_{0 q \mid L}^{\left(j_{L}, j_{R} \mid 0\right)}=\alpha_{q \mid L}^{\left(j_{L}, j_{R} \mid 0\right)}, \\
& \alpha_{s q \mid r_{k} h_{k}}^{\left(j_{L}, j_{R} \mid J\right)\left(R_{k}, H_{k}\right)}=\delta_{s r_{k}} \alpha_{q \mid h_{k}}^{\left(j_{L}, j_{R} \mid J\right)\left(R_{k}, H_{k}\right)}, \quad \text { if } \quad\left(j_{L}, j_{R}\right) \supset R_{k}, \\
& \chi_{a, s q \mid b}^{\left(j_{L}, j_{R} \mid J\right)}=<1 a, 1 b \mid J s>\chi_{q}^{\left(j_{L}, j_{R} \mid J\right)}, J=0,1,2, \\
& \chi_{a, s q \mid L}^{\left(j_{L}, j_{R} \mid 1\right)}=\delta_{a s} \chi_{q \mid L}^{\left(j_{L}, j_{R} \mid 1\right)}, \\
& \chi_{a, s q \mid r_{k} h_{k}}^{\left(j_{L}, j_{R} \mid J\right)\left(R_{k}, H_{k}\right)}=<1 a, R_{k} r_{k} \mid J s>\chi_{q \mid h_{k}}^{\left(j_{L}, j_{R} \mid J\right)\left(R_{k}, H_{k}\right)} .
\end{aligned}
$$

In the last equation $J$ takes the values $j_{k}-1, j_{k}, j_{k}+1$, where $j_{k}$ is the angular momentum describing the representation $R_{k}$. We conclude that the possible values of $J$ 
which determine the degree of harmonics which appear in the harmonic expansion are $\left\{0,1,2, j_{k}-1, j_{k}, j_{k}+1\right\}$. In particular:

$$
J_{\max }=\max \left(2, j_{\max }+1\right)
$$

where $j_{\text {max }}$ is the maximum angular momentum corresponding to the representations $R_{k}$ which appears in the decomposition (32) of $G$ under $S O(3)$. The harmonic expansions read :

$$
\begin{aligned}
A_{0}(t, y)= & \sum_{\left(j_{L}, j_{R}\right) \supset 1} \sum_{b, q} D_{b q}^{\left(j_{L}, j_{R} \mid 1\right)}(L(y)) \alpha_{q}^{\left(j_{L}, j_{R} \mid 1\right)}(t) T_{b} \\
+ & \sum_{\left(j_{L}, j_{R}\right) \supset 0} \sum_{q, L} D_{0 q}^{\left(j_{L}, j_{R} \mid 0\right)}(L(y)) \alpha_{q \mid L}^{\left(j_{L}, j_{R} \mid 0\right)}(t) T_{L} \\
+ & \sum_{\left(R_{k}, H_{k}\right)} \sum_{q,\left(j_{L}, j_{R}\right) \supset R_{k}} D_{r_{k} q}^{\left(j_{L}, j_{R} \mid R_{k}\right)}(L(y)) \alpha_{q \mid h_{k}}^{\left(j_{L}, j_{R} \mid R_{k}\right)\left(R_{k}, H_{k}\right)}(t) T_{r_{k} h_{k}} \\
A_{a}(t, y)= & A_{a}^{B} \\
+ & \sum_{J=0,1,2} \sum_{\left(j_{L}, j_{R}\right) \supset J} \sum_{b, p, q} D_{p q}^{\left(j_{L}, j_{R} \mid J\right)}(L(y))<1 a, 1 b \mid J p>\chi_{q}^{\left(j_{L}, j_{R} \mid J\right)}(t) T_{b} \\
+ & \sum_{\left(j_{L}, j_{R}\right) \supset 1} \sum_{q, L} D_{a q}^{\left(j_{L}, j_{R} \mid 1\right)}(L(y)) \chi_{q \mid L}^{\left(j_{L}, j_{R} \mid 1\right)}(t) T_{L} \\
+ & \sum_{\left(R_{k}, H_{k}\right)} \sum_{J=R_{k}-1, R_{k}, R_{k}+1} \sum_{\left(j_{L}, j_{R}\right) \supset J} \sum_{p, q, r_{k}, h_{k}} D_{p q}^{\left(j_{L}, j_{R} \mid J\right)}(L(y)) \\
& <1 a, R_{k} r_{k} \mid J p>\chi_{q \mid h_{k}}^{\left(j_{L}, j_{R} \mid J\right)\left(R_{k} H_{k}\right)}(t) T_{r_{k} h_{k}} .
\end{aligned}
$$

The expression $D_{0 q}^{\left(j_{L}, j_{R} \mid 0\right)}(L(y))$ on the second line of (42) just means that the first index of this harmonic takes the value corresponding to the identity representation in the product $\left(j_{L}, j_{R}\right)$. On the other hand the notation $\sum_{\left(j_{L}, j_{R}\right) \supset J}$ means that we sum over all the tensor product representations $\left(j_{L}, j_{R}\right)$ of $S O(4) \approx S U(2)_{L} \otimes S U(2)_{R}$, which contain the representation $J$ upon restriction to $S U(2)_{\text {diag }}$, as described in appendix B.

If the gauge group $G$ is $S O(N)$, several simplifications occur. The decomposition of the adjoint of $S O(N)$ under $R=S O(3)$ can be written in a more concrete form and it is given in eq. (14). The commutation relations of the generators, heavily used in the calculations, corresponding to the decomposition (14) can be found in eqs. (15).

Maybe the most important simplification brought about is that the only representation appearing in the decomposition (14) is the triplet, corresponding to angular momentum $j_{\max }=1$. This makes things much easier, since according to the statements made above only scalar- vector- and tensor-harmonics appear in the expansion. In this case we are also in a position to give explicit expressions for the relevant Clebsch-Gordan coefficients. 
Changing from the $S U(2)$ standard basis to the "tensor" basis they read :

$$
\begin{array}{lll}
<1 a, 1 b \mid 00> & \rightarrow & \delta_{a b} \\
<1 a, 1 b \mid 1 p> & \rightarrow & \varepsilon_{a b p} \\
<1 a, 1 b \mid 2 p_{1} p_{2}> & \rightarrow & \frac{1}{2}\left(\delta_{a p_{1}} \delta_{b p_{2}}+\delta_{b p_{1}} \delta_{a p_{2}}\right)-\frac{1}{3} \delta_{a b} \delta_{p_{1} p_{2}} .
\end{array}
$$

All indices run from 1 to 3 and we have represented the $J=2$ components by the double index $p_{1} p_{2}$, as in Appendix B.

We are going to discuss in the following section the case $N=3$. Most of the physical features of the model are present in this case; on the other hand, calculations are much easier, because of some additional simplifications. Also the formulae to be presented, although far from being simple, are much more economical. The simplifications coming from choosing $N=3$ are summarized below.

One may see that the parts of the expansion having to do with adj $S O(N-3)$ and $\left(R_{k}, H_{k}\right)$ (cf. eq. (32)) no longer exist. Thus the harmonic expansion becomes :

$$
\begin{aligned}
A_{0}(t, y)= & \sum_{\left(j_{L}, j_{R}\right) \supset 1} \sum_{b, q} D_{b q}^{\left(j_{L}, j_{R} \mid 1\right)}(L(y)) \alpha_{q}^{\left(j_{L}, j_{R} \mid 1\right)}(t) T_{b}, \\
A_{a}(t, y)= & A_{a}^{B}+\sum_{J=0,1,2} \sum_{\left(j_{L}, j_{R}\right) \supset J} \sum_{b, p, q} D_{p q}^{\left(j_{L}, j_{R} \mid J\right)}(L(y)) \\
& <1 a, 1 b \mid J p>\chi_{q}^{\left(j_{L}, j_{R} \mid J\right)}(t) T_{b} .
\end{aligned}
$$

We separate the background field and the $y$-independent part in the last equation and write explicitly the sum over $J$ :

$$
\begin{aligned}
A_{a}(t, y) & =\left(1+\chi_{0}(t)\right) T_{a} \\
& +\sum_{\left(j_{L}, j_{R}\right) \supset 0} \sum_{b, q} D_{0 q}^{\left(j_{L}, j_{R} \mid 0\right)}(L(y)) \delta_{a b} \chi_{q}^{\left(j_{L}, j_{R} \mid 0\right)}(t) T_{b} \\
& +\sum_{\left(j_{L}, j_{R}\right) \supset 1} \sum_{b, p, q} D_{p q}^{\left(j_{L}, j_{R} \mid 1\right)}(L(y)) \varepsilon_{a b p} \chi_{q}^{\left(j_{L}, j_{R} \mid 1\right)}(t) T_{b} \\
& +\sum_{\left(j_{L}, j_{R}\right) \supset 2} \sum_{b, q, p_{1}, p_{2}}\left[\frac{1}{2}\left(\delta_{a p_{1}} \delta_{b p_{2}}+\delta_{b p_{1}} \delta_{a p_{2}}\right)\right. \\
& \left.-\frac{1}{3} \delta_{a b} \delta_{p_{1} p_{2}}\right] D_{\left(p_{1} p_{2}\right) q}^{\left(j_{L}, j_{R} \mid 2\right)}(L(y)) \chi_{q}^{\left(j_{L}, j_{R} \mid 2\right)}(t) T_{b},
\end{aligned}
$$

where we have plugged in the explicit expressions (44) for the Clebsch-Gordan coefficients. The Clebsch-Gordan coefficient $\frac{1}{2}\left(\delta_{a p_{1}} \delta_{b p_{2}}+\delta_{b p_{1}} \delta_{a p_{2}}\right)-\frac{1}{3} \delta_{a b} \delta_{p_{1} p_{2}}$ in fact coincides with the operator $P\left(p_{1} p_{2} \mid q_{1} q_{2}\right)$, which projects out the part of the harmonic $D_{\left(p_{1} p_{2}\right) q}^{\left(j_{L}, j_{2} \mid 2\right)}(L(y))$ which is symmetric and traceless in the indices $p_{1}$ and $p_{2}$. Thus, this Clebsch-Gordan coefficient just projects out the harmonics $G_{a b \mid q}^{(n \pm)}(L(y)), S_{a b \mid q}^{(n \pm)}(L(y))$ and $P_{a b \mid q}^{(n)}(L(y))$, discussed in 
Appendix B, where it is also explained that the harmonics $D_{a q}^{\left(j_{L}, j_{R} \mid 1\right)}(L(y))$ will give rise to $S_{a \mid q}^{(n \pm)}(L(y))$ and $P_{a \mid q}^{(n)}(L(y))$. Similarly, the harmonics $D_{0 q}^{\left(j_{L}, j_{R} \mid 0\right)}(L(y))$ become $Q_{q}^{(n)}(L(y))$. Then, introducing some numerical factors for later convenience, the final form of the harmonic expansion in this case reads

$$
\begin{aligned}
A_{0}(t, y) & =\sum_{n} \sum_{b, q}\left[\sqrt{2} \alpha_{q}^{(n+)}(t) S_{b \mid q}^{(n+)}(L(y))+\sqrt{2} \alpha_{q}^{(n-)}(t) S_{b \mid q}^{(n-)}(L(y))\right. \\
& \left.+\frac{1}{\sqrt{6}} \beta_{q}^{(n)}(t) P_{b \mid q}^{(n)}(L(y))\right] T_{b} \\
A_{a}(t, y) & =A_{a}^{(0)} \\
& +\sum_{n} \sum_{b, q} \frac{1}{\sqrt{6}} \gamma_{q}^{(n)}(t) \delta_{a b} Q_{q}^{(n)}(L(y)) T_{b} \\
& +\left[\sum_{n} \sum_{b, q} \frac{1}{\sqrt{2}} \rho_{q}^{(n+)}(t) S_{c \mid q}^{(n+)}(L(y))+\frac{1}{\sqrt{2}} \rho_{q}^{(n-)}(t) S_{c \mid q}^{(n-)}(L(y))\right. \\
& \left.+\sum_{n} \sum_{b, q} \frac{1}{\sqrt{6}} \sigma_{q}^{(n)}(t) P_{c \mid q}^{(n)}(L(y))\right] \varepsilon_{a c b} T_{b} \\
& +\sum_{n} \sum_{b, q}\left[\mu_{q}^{(n+)}(t) G_{a b \mid q}^{(n+)}(L(y))+\mu_{q}^{(n-)}(t) G_{a b \mid q}^{(n-)}(L(y))\right] T_{b} \\
& +\sum_{n} \sum_{b, q} \frac{1}{\sqrt{2}}\left[\nu_{q}^{(n+)}(t) S_{a b \mid q}^{(n+)}(L(y))+\nu_{q}^{(n-)}(t) S_{a b \mid q}^{(n-)}(L(y))\right] T_{b} \\
& +\sum_{n} \sum_{b, q} \sqrt{6}\left[\xi_{q}^{(n)}(t) P_{a b \mid q}^{(n)}(L(y))\right] T_{b},
\end{aligned}
$$

with

$$
A_{a}^{(0)}=\left(1+\chi_{0}(t)\right) T_{a}
$$

\section{The Perturbed Model}

Let us now consider inhomogeneous perturbations around our minisuperspace. We use the exact action in terms of the background quantities but expand only to second order in the perturbations. We assume that the metric is of the form given in eq. (四) except that it is multiplied with the normalization factor $\sigma^{2}$. The gravitational part of our model is treated in exactly the same way as in ref. [3]. Therefore let us recall that

$$
h_{a b}=\sigma^{2} e^{2 \alpha}\left(\delta_{a b}+\varepsilon_{a b}\right),
$$

where $e^{\alpha}=a$ and (in simplified notation)

$$
\varepsilon_{a b}=\frac{\sqrt{6}}{3} \delta_{a b} a_{n} Q^{n}+\sqrt{6} b_{n} P_{a b}^{n}+\sqrt{2} c_{n} S_{a b}^{n}+2 d_{n} G_{a b}^{n} .
$$

Furthermore for the lapse and shift functions we have :

$$
N=N_{0}\left[1+\frac{1}{\sqrt{6}} g_{n} Q^{n}\right],
$$




$$
N_{a}=e^{\alpha}\left[\frac{1}{\sqrt{6}} k_{n} P_{a}^{n}+\sqrt{2} j_{n} S_{a}^{n}\right] .
$$

Let us remark here that, of course, these expansions are exactly the same as the ones we get, if we apply the symmetric field constraints on the functions $\varepsilon_{a b}, N$ and $N_{a}$. Now the gravitational part of the action (5) takes the form [3]

$$
S_{G R}=S_{G R_{0}}+V_{S^{3}} \sum_{n} \int d t L_{G R}^{n}
$$

where

$$
\begin{aligned}
& L_{G R}^{n}=\frac{1}{2} e^{\alpha} N_{0} \quad\left[\frac{1}{3}\left(n^{2}-\frac{5}{2}\right) a_{n}^{2}+\frac{n^{2}-7}{3} \frac{n^{2}-4}{n^{2}-1} b_{n}^{2}-2\left(n^{2}-4\right) c_{n}^{2}\right. \\
& -\left(n^{2}+1\right) d_{n}^{2}+\frac{2}{3}\left(n^{2}-4\right) a_{n} b_{n}+\frac{2}{3}\left(n^{2}-4\right) g_{n} b_{n} \\
& \left.+\frac{2}{3}\left(n^{2}+\frac{1}{2}\right) g_{n} a_{n}\right] \\
& +2 \frac{e^{\alpha}}{N_{0}} \quad\left[-\frac{1}{3\left(n^{2}-1\right)} k_{n}^{2}+\left(n^{2}-4\right) j_{n}^{2}\right] \\
& +\frac{1}{2} \frac{e^{3 \alpha}}{N_{0}} \quad\left\{-\dot{a}_{n}^{2}+\frac{n^{2}-4}{n^{2}-1} \dot{b}_{n}^{2}+\left(n^{2}-4\right) \dot{c}_{n}^{2}+\dot{d}_{n}^{2}\right. \\
& +\dot{\alpha}\left[-2 a_{n} \dot{a}_{n}+8 \frac{n^{2}-4}{n^{2}-1} b_{n} \dot{b}_{n}+8\left(n^{2}-4\right) c_{n} \dot{c}_{n}+8 d_{n} \dot{d}_{n}+2 g_{n} \dot{a}_{n}\right] \\
& +\dot{\alpha}^{2}\left[-\frac{3}{2} a_{n}^{2}+6 \frac{n^{2}-4}{n^{2}-1} b_{n}^{2}+6\left(n^{2}-4\right) c_{n}^{2}+6 d_{n}^{2}+3 a_{n} g_{n}-g_{n}^{2}\right] \\
& \left.+e^{-\alpha}\left[k_{n}\left(-\frac{2}{3} \dot{a}_{n}-\frac{2}{3} \frac{n^{2}-4}{n^{2}-1} \dot{b}_{n}+\frac{2}{3} \dot{\alpha} g_{n}\right)-2\left(n^{2}-4\right) \dot{c}_{n} j_{n}\right]\right\} \\
& -3 e^{3 \alpha} N_{0} \quad H^{2}\left[\frac{1}{4} a_{n}^{2}-\frac{n^{2}-4}{n^{2}-1} b_{n}^{2}-\left(n^{2}-4\right) c_{n}^{2}-d_{n}^{2}+\frac{1}{2} a_{n} g_{n}\right] \text {. }
\end{aligned}
$$

For the YM part of the action with gauge group $G=S O(3)$ we write the field strength as

$$
\begin{aligned}
& F_{a 0}=F_{a 0}^{(0)}+\nabla_{a}^{(0)} \bar{A}_{0}-\nabla_{0}^{(0)} \bar{A}_{a}+\left[\bar{A}_{a}, \bar{A}_{0}\right], \\
& F_{a b}=F_{a b}^{(0)}+\nabla_{a}^{(0)} \bar{A}_{b}-\nabla_{b}^{(0)} \bar{A}_{a}+\left[\bar{A}_{a}, \bar{A}_{b}\right],
\end{aligned}
$$

with the symmetric field $F^{(0)}$ given in eq. (17) and a covariant derivative with respect to the symmetric field :

$$
\nabla_{a}^{(0)}=\nabla_{a}+\left[A_{a}^{(0)}, \cdot\right], \quad \nabla_{0}^{(0)}=\partial_{0} .
$$

Inserting the symmetric field (13) and the harmonic expansions (48),(49) together with 
the above expressions in eq. (7) we obtain

$$
S_{Y M}=S_{Y M_{0}}+\frac{V_{S^{3}}}{e^{2}} \sum_{n} \int d t L_{Y M}^{n},
$$

with

$$
L_{Y M}^{n}=\frac{N_{0}}{e^{\alpha}} \mathcal{L}_{1}^{n}+\frac{e^{\alpha}}{N_{0}} \mathcal{L}_{2}^{n}+\frac{1}{N_{0} e^{\alpha}} \mathcal{L}_{3}^{n}+\frac{1}{N_{0}} \mathcal{L}_{4}^{n}
$$

and

$$
\begin{aligned}
& \mathcal{L}_{1}=\left(\chi_{0}^{2}-1\right)^{2}\left(-\frac{3}{8} a_{n}^{2}+\frac{1}{4} a_{n} g_{n}-\frac{3}{2} \frac{n^{2}-4}{n^{2}-1} b_{n}^{2}-\frac{3}{2} d_{n}^{2}-\frac{3}{2}\left(n^{2}-4\right) c_{n}^{2}\right) \\
& +\chi_{0}\left(\chi_{0}^{2}-1\right)\left(a_{n} \gamma_{n}+4 \frac{n^{2}-4}{n^{2}-1} b_{n} \xi_{n}+2\left(n^{2}-4\right) c_{n} \nu_{n}+2 d_{n} \mu_{n}-g_{n} \gamma_{n}\right) \\
& +\chi_{0}^{2}\left(-\frac{3}{2} \gamma_{n}^{2}-\rho_{n}^{2}-\frac{1}{3} \frac{1}{n^{2}-1} \sigma_{n}^{2}+\frac{1}{3} a_{n} \sigma_{n}-\frac{2}{3} \frac{n^{2}-4}{n^{2}-1} b_{n} \sigma_{n}\right. \\
& \left.-\left(n^{2}-4\right) c_{n} \rho_{n}-\frac{1}{3} g_{n} \sigma_{n}+n\left(n^{2}-4\right) \tilde{c}_{n} \nu_{n}+2 n \tilde{d}_{n} \mu_{n}\right) \\
& +\chi_{0}\left(-\gamma_{n} \sigma_{n}+\frac{1}{2} n \tilde{\rho}_{n} \rho_{n}-n \tilde{\mu}_{n} \mu_{n}-\frac{1}{2} n\left(n^{2}-4\right) \tilde{\nu}_{n} \nu_{n}\right) \\
& -\frac{1}{6}\left(n^{2}-4\right) \gamma_{n}^{2}-\frac{1}{4}\left(n^{2}-4\right) \rho_{n}^{2}-\frac{1}{6} \frac{n^{2}-3}{n^{2}-1} \sigma_{n}^{2}-\frac{1}{4}\left(n^{2}-4\right) n^{2} \nu_{n}^{2} \\
& -\frac{1}{2}\left(n^{2}+1\right) \mu_{n}^{2}-\frac{2}{3}\left(n^{2}-4\right) \xi_{n}^{2}-\frac{1}{3} a_{n} \sigma_{n}+\frac{2}{3} \frac{n^{2}-4}{n^{2}-1} b_{n} \sigma_{n}+\left(n^{2}-4\right) c_{n} \rho_{n} \\
& +\frac{1}{3} g_{n} \sigma_{n}-n\left(n^{2}-4\right) \tilde{c}_{n} \nu_{n}-2 n \tilde{d}_{n} \mu_{n}-\frac{2}{3}\left(n^{2}-4\right) \gamma_{n} \xi_{n}+\frac{1}{2} n\left(n^{2}-4\right) \tilde{\rho}_{n} \nu_{n} \text {, } \\
& \mathcal{L}_{2}=\chi_{0}^{2}\left(2 \alpha_{n}^{2}+\frac{1}{6} \frac{1}{n^{2}-1} \beta_{n}^{2}\right)+\chi_{0}\left(-2 \alpha_{n} \dot{\rho_{n}}-\frac{1}{3} \frac{1}{n^{2}-1} \beta_{n} \dot{\sigma_{n}}+2 n \tilde{\alpha}_{n} \alpha_{n}\right) \\
& +\dot{\chi}_{0}^{2}\left(-\frac{1}{8} a_{n}^{2}+\frac{1}{2} \frac{n^{2}-4}{n^{2}-1} b_{n}^{2}+\frac{1}{2}\left(n^{2}-4\right) c_{n}^{2}+\frac{1}{2} d_{n}^{2}+\frac{1}{4} g_{n}^{2}-\frac{1}{4} a_{n} g_{n}\right) \\
& +\dot{\chi}_{0}\left(\frac{1}{6} a_{n} \beta_{n}+\frac{1}{2} a_{n} \dot{\gamma}_{n}+\frac{2}{3} \frac{n^{2}-4}{n^{2}-1} b_{n} \beta_{n}-4 \frac{n^{2}-4}{n^{2}-1} b_{n} \dot{\xi}_{n}+2\left(n^{2}-4\right) c_{n} \alpha_{n}\right. \\
& \left.-2\left(n^{2}-4\right) c_{n} \dot{\nu_{n}}-2 d_{n} \dot{\mu_{n}}-\frac{1}{6} g_{n} \beta_{n}-\frac{1}{2} g_{n} \dot{\gamma_{n}}+2 \alpha_{n} \rho_{n}+\frac{1}{3} \frac{1}{n^{2}-1} \beta_{n} \sigma_{n}\right) \\
& +\left(n^{2}-2\right) \alpha_{n}^{2}-\left(n^{2}-4\right) \alpha_{n} \dot{\nu_{n}}+\frac{1}{12} \frac{n^{2}-3}{n^{2}-1} \beta_{n}^{2}+\frac{1}{6} \beta_{n} \dot{\gamma_{n}}-\frac{2}{3} \frac{n^{2}-4}{n^{2}-1} \beta_{n} \dot{\xi_{n}} \\
& +\frac{1}{4}{\dot{\gamma_{n}}}^{2}+\frac{1}{2}{\dot{\rho_{n}}}^{2}+\frac{1}{6} \frac{1}{n^{2}-1}{\dot{\sigma_{n}}}^{2}+\frac{1}{2}{\dot{\mu_{n}}}^{2}+\frac{1}{2}\left(n^{2}-4\right){\dot{\nu_{n}}}^{2}+2 \frac{n^{2}-4}{n^{2}-1} \dot{\xi}_{n}{ }^{2}-n \tilde{\alpha}_{n}{\dot{\rho_{n}}}, \\
& \mathcal{L}_{3}=\left(\chi_{0}^{2}-1\right)^{2}\left(\frac{1}{6\left(n^{2}-1\right)} k_{n}^{2}+2 j_{n}^{2}\right),
\end{aligned}
$$

${ }^{3}$ Here we give the Minkowskian action. For the Euclidean version the signs of $\mathcal{L}_{2}^{n}$ and $\mathcal{L}_{3}^{n}$ have to be reversed. 


$$
\begin{aligned}
\mathcal{L}_{4}= & \chi_{0} \dot{\chi}_{0}\left(-\frac{1}{3} \frac{1}{n^{2}-1} k_{n} \sigma_{n}-2 j_{n} \rho_{n}\right)+\chi_{0}\left(\chi_{0}^{2}-1\right)\left(-\frac{1}{3} \frac{1}{n^{2}-1} k_{n} \beta_{n}-4 j_{n} \alpha_{n}\right) \\
& +\dot{\chi}_{0}\left(-\frac{1}{3} k_{n} \gamma_{n}-\frac{2}{3} \frac{n^{2}-4}{n^{2}-1} k_{n} \xi_{n}-\left(n^{2}-4\right) j_{n} \nu_{n}+n \tilde{j}_{n} \rho_{n}\right) \\
& +\left(\chi_{0}^{2}-1\right)\left(\frac{1}{3} \frac{1}{n^{2}-1} k_{n} \dot{\sigma}_{n}+2 j_{n} \dot{\rho}_{n}-2 n \tilde{j}_{n} \alpha_{n}\right) .
\end{aligned}
$$

In these (and the following) formulae a sum over the even and the odd part of the relevant coefficients is implied. The tilde over a coefficient signals an off-diagonal term connecting even and odd perturbations with each other f: $\tilde{p}_{n}^{(\text {even/odd })}=p_{n}^{(\text {odd/even })}$ for a generic coefficient $p_{n}$.

One may ask about the remnants of gauge symmetry in this Lagrangian. To answer this question we start with an ordinary infinitesimal gauge transformation $\delta A_{\mu}=\nabla_{\mu} U-$ $\left[U, A_{\mu}\right], U=u_{a} T_{a}$ and demand that the symmetric field $A^{(0)}$ remains fixed. The gauge transformation will therefore exclusively act on the expansion $\bar{A}$ and we find $\delta A_{\mu}=\nabla_{\mu}^{(0)} U$ in the leading order. Expanding the parameter $u_{i}$

$$
u_{a}=\frac{1}{\sqrt{6}} v_{n} P_{a}^{n}+\sqrt{2} w_{n} S_{a}^{n}
$$

and using the expression (13) for the symmetric field as well as eqs. (48) and (49) for $\bar{A}$ this transformation property can be translated to the coefficients :

$$
\begin{aligned}
& \delta \gamma_{n}=-\frac{1}{3} v_{n}, \quad \delta \xi_{n}=\frac{1}{6} v_{n}, \quad \delta \sigma_{n}=\chi_{0} v_{n}, \quad \delta \beta_{n}=\dot{v}_{n}, \\
& \delta \nu_{n}=w_{n}, \quad \delta \alpha_{n}=\dot{w}_{n}, \quad \delta \rho_{n}=2 \chi_{0} w_{n}+n \tilde{w}_{n}, \quad \delta \mu_{n}=0 .
\end{aligned}
$$

Our procedure is analogous to the definition of coordinate transformations acting on the perturbations of a certain background metric as given in ref. [14]. This analogy can be pushed one step further by introducing gauge invariant variables

$$
\begin{aligned}
& \Gamma_{n}=\gamma_{n}+2 \xi_{n}, \quad B_{n}=\beta_{n}+3 \dot{\gamma}_{n}, \quad S_{n}=\sigma_{n}+3 \chi_{0} \gamma_{n}, \\
& A_{n}=\alpha_{n}-\dot{\nu}_{n} \quad R_{n}=\rho_{n}-2 \chi_{0} \nu_{n}-n \tilde{\nu}_{n} .
\end{aligned}
$$

As in the case of coordinate transformations in pure gravity the tensor mode $\mu$ is invariant, since the expansion (65) does not contain any tensor degree of freedom. It might be

\footnotetext{
${ }^{4}$ This mixing of the odd and even parts may give rise to questions about parity conservation when the perturbations are included. This does not create any problem though: If we start from the parity transformation properties of the metric and the gauge fields, we may derive the ones of $N_{0}(t), a(t), \chi_{0}(t)$ and the perturbation coefficients. Using these properties, it turns out that the second-order Lagrangian is parity invariant.
} 
interesting to note that the above variables represent a linearized version of the invariant quantities $\operatorname{tr}\left(B_{c} B_{d}\right)$ introduced by Lüscher [15] ( $B_{c}=\epsilon_{a c b} F_{a b}$ is the magnetic field.). With these variables one gets, for instance:

$$
R_{n}=\frac{\sqrt{2}}{8\left(n^{2}-4\right)\left(\chi_{0}^{2}-1\right)} \int d \mu(y) \operatorname{tr}\left(B_{c} B_{d}\right) S_{c d}^{n} .
$$

The important observation is now that our Lagrangian (60) is invariant under the transformations (66) provided the field $\chi_{0}$ fulfills the background equation of motion to be derived from the action (18). Consequently, in such a case the Lagrangian can be written in terms of the gauge invariant variables only. For a special solution $\chi_{0}$ we will demonstrate this explicitly in section 5 .

One can proceed from this point by defining the conjugate momenta and obtaining the Hamiltonian in the usual manner. Here we want to indicate the structure of the Hamiltonian

$$
H=N_{0}\left(\mathcal{H}_{0}+\sum_{n} \mathcal{H}_{2}^{(n)}+\sum_{n} g_{n} \mathcal{H}_{g}^{(n)}\right)+\sum_{n}\left(k_{n} \mathcal{H}_{k}^{(n)}+j_{n} \mathcal{H}_{j}^{(n)}\right)+\sum_{n}\left(\alpha_{n} \mathcal{H}_{\alpha}^{(n)}+\beta_{n} \mathcal{H}_{\beta}^{(n)}\right)
$$

which we find in accordance with eq. (8). The linear YM gauge constraints $\mathcal{H}_{\alpha}^{(n)}$ and $\mathcal{H}_{\beta}^{(n)}$ responsible for the gauge invariance of the wave function read

$$
\begin{aligned}
& \mathcal{H}_{\alpha}^{(n)}=2 \chi_{0} \pi_{\rho_{n}}-\frac{2}{3} \rho_{n} \pi_{\chi}+\pi_{\nu_{n}}+n \tilde{\pi}_{\rho_{n}}, \\
& \mathcal{H}_{\beta}^{(n)}=\chi_{0} \pi_{\sigma_{n}}-\frac{1}{9\left(n^{2}-1\right)} \sigma_{n} \pi_{\chi}-\frac{1}{3} \pi_{\gamma_{n}}+\frac{1}{6} \pi_{\xi_{n}},
\end{aligned}
$$

with the conjugate momenta

$$
\begin{aligned}
& \pi_{\chi}=\frac{3 V_{S^{3}} e^{\alpha}}{e^{2} N_{0}} \dot{\chi}_{0}+(\text { quadratic terms }), \\
& \pi_{\gamma_{n}}=\frac{V_{S^{3}} e^{\alpha}}{2 e^{2} N_{0}}\left[\dot{\gamma}_{n}+\frac{1}{3} \beta_{n}+\dot{\chi}_{0}\left(a_{n}-g_{n}\right)\right], \\
& \pi_{\rho_{n}}=\frac{V_{S^{3}} e^{\alpha}}{e^{2} N_{0}}\left[\dot{\rho}_{n}-2 \chi_{0} \alpha_{n}-n \tilde{\alpha}_{n}\right]+\frac{2}{e^{2} N_{0}}\left(\chi_{0}^{2}-1\right) j_{n}, \\
& \pi_{\sigma_{n}}=\frac{V_{S^{3}} e^{\alpha}}{3 e^{2} N_{0}\left(n^{2}-1\right)}\left[\dot{\rho}_{n}-\chi_{0} \beta_{n}\right]+\frac{1}{3 e^{2} N_{0}\left(n^{2}-1\right)}\left(\chi_{0}^{2}-1\right) k_{n}, \\
& \pi_{\mu_{n}}=\frac{V_{S^{3}} e^{\alpha}}{e^{2} N_{0}}\left[\dot{\mu}_{n}-2 \dot{\chi}_{0} d_{n}\right] \\
& \pi_{\nu_{n}}=\frac{V_{S^{3}} e^{\alpha}\left(n^{2}-4\right)}{e^{2} N_{0}}\left[\dot{\nu}_{n}-\alpha_{n}-2 \dot{\chi}_{0} c_{n}\right], \\
& \pi_{\xi_{n}}=\frac{4 V_{S^{3}} e^{\alpha}\left(n^{2}-4\right)}{e^{2} N_{0}\left(n^{2}-1\right)}\left[\dot{\xi}_{n}-\frac{1}{6} \beta_{n}-\dot{\chi}_{0} b_{n}\right] .
\end{aligned}
$$

Their quantized version can be used to generate our gauge transformations (66) performing the commutator $\delta A_{a}=\left[A_{a}, w_{n} \mathcal{H}_{\alpha}^{(n)}+v_{n} \mathcal{H}_{\beta}^{(n)}\right]$ with the harmonic expansion (49)

\footnotetext{
${ }^{5}$ We thank C. Lee for pointing out this relation.
} 
for $A_{a}$. The pure gravitational parts of eq. (69) and the momenta for the gravitational perturbations can be found in ref. [3]. We will not give the other YM parts explicitly since we are mainly interested in the semi-classical wave function in a region of superspace where the minisuperspace variables are classical but the perturbations are quantum mechanical. Therefore, it is enough in our approximation to solve first the Euclidean background equations by setting the perturbations equal to zero and then solve the equations of motion for the perturbations subject to the Hartle-Hawking boundary condition by neglecting their back reactions on the background. In this way, the wave function is given by $\Psi=C \exp \left(-\hat{I}_{E}\right)$ where $C$ is a semi-classical prefactor and $\hat{I}_{E}$ the extremal Euclidean action. $\hat{I}_{E}$ is given by $\hat{I}_{E}=(1 / 2) p_{n} \pi_{p_{n}}$ where $p_{n}$ is a generic perturbation.

\section{The Wave Function}

Let us follow the above program for the particular case of the EYM system which we are considering here. The background equations of motion for the minisuperspace degrees of freedom $\alpha, \chi_{0}$ are given by

$$
\begin{gathered}
e^{3 \alpha} \frac{d}{d t}\left(\frac{\dot{\alpha}}{N_{0}}\right)+\frac{3}{2} \frac{e^{3 \alpha}}{N_{0}} \dot{\alpha}^{2}+\frac{1}{2} N_{0} e^{\alpha}-\frac{3}{2} H^{2} N_{0} e^{3 \alpha}+\frac{1}{2 e^{2}}\left(\frac{e^{\alpha}}{2 N_{0}} \dot{\chi}_{0}^{2}+\frac{N_{0}}{e^{\alpha}} V\right)=0, \\
\frac{e^{\alpha}}{N_{0}} \frac{d}{d t}\left(\frac{e^{\alpha}}{N_{0}} \dot{\chi}_{0}\right)+V^{\prime}=0
\end{gathered}
$$

while for $N_{0}$ we obtain the constraint

$$
\frac{e^{3 \alpha}}{2}\left(\frac{\dot{\alpha}^{2}}{N_{0}^{2}}+e^{-2 \alpha}-H^{2}\right)-\frac{1}{2 e^{2} N_{0}}\left(\frac{e^{\alpha}}{2 N_{0}} \dot{\chi}_{0}^{2}+\frac{N_{0}}{e^{\alpha}} V\right)=0 .
$$

Here $V^{\prime}$ denotes the derivative of $V=1 / 2\left(1-\chi_{0}^{2}\right)^{2}$ (cf. eq. (20)) with respect to $\chi_{0}$. The perturbations of the gravitational and YM fields can be separated into three types, namely tensor $\left(d_{n}, \mu_{n}\right)$, vector $\left(c_{n}, \nu_{n}, j_{n}, \alpha_{n}, \rho_{n}\right)$ and scalar $\left(a_{n}, b_{n}, \sigma_{n}, \gamma_{n}, \xi_{n}, \beta_{n}, g_{n}, k_{n}\right)$ modes. In the equations of motion different types of perturbations do not couple. This observation allows to consider separately each type of perturbation and calculate their corresponding contribution to the wave function.

For the tensor perturbations $d_{n}$ and $\mu_{n}$ we obtain the following equations of motion

$$
\begin{aligned}
\frac{d}{d t}\left(\frac{e^{3 \alpha}}{N_{0}} \dot{d}_{n}\right)+e^{\alpha} N_{0}\left(n^{2}-1\right) d_{n}= & \frac{N_{0}}{e^{2} e^{\alpha}}\left[4\left(\frac{e^{2 \alpha}}{2 N_{0}^{2}} \dot{\chi}_{0}^{2}-V\right) d_{n}+V^{\prime} \mu_{n}+2 n U \tilde{\mu}_{n}\right. \\
& \left.-2 \frac{e^{2 \alpha}}{N_{0}^{2}} \dot{\chi}_{0} \dot{\mu}_{n}\right] \\
\frac{e^{\alpha}}{N_{0}} \frac{d}{d t}\left(\frac{e^{\alpha}}{N_{0}} \dot{\mu}_{n}\right)+\left(n^{2}+1\right) \mu_{n}= & 2 \frac{e^{2 \alpha}}{N_{0}^{2}} \dot{\chi}_{0} \dot{d}_{n}-2 n \chi_{0} \tilde{\mu}_{n}+2 n U \tilde{d}_{n}-V^{\prime} d_{n}
\end{aligned}
$$


with the abbreviation $U=\chi_{0}^{2}-1$.

Let us now consider the case of the vector perturbations. To simplify the calculation one can make a choice of gauge. For the gravitational field we choose to proceed in the transverse-traceless gauge which corresponds for the vector modes in $c_{n}=0$. For the YM field inspection of the transformations (66) shows that we are free to take $\rho_{n}=0$. In this gauge the equations of motion and the constraints of the remaining variables $\left(\nu_{n}, j_{n}, \alpha_{n}\right)$ are given by :

$$
\begin{aligned}
\frac{e^{\alpha}}{N_{0}} \frac{d}{d t}\left(\frac{e^{\alpha}}{N_{0}}\left(\dot{\nu}_{n}-\alpha_{n}\right)\right)+\frac{1}{2} n^{2} \nu_{n} & =-\chi_{0} n \tilde{\nu}_{n}-\frac{e^{\alpha}}{N_{0}^{2}} j_{n} \dot{\chi}_{0} \\
{\left[e^{\alpha}\left(n^{2}-4\right)+\frac{2}{e^{2} e^{\alpha}} V\right] j_{n} } & =\frac{1}{4 e^{2}}\left(2 V^{\prime} \alpha_{n}+\dot{\chi}_{0}\left(n^{2}-4\right) \nu_{n}+2 n U \tilde{\alpha}_{n}\right), \\
e^{\alpha}\left[2\left(n^{2}+2 U\right) \alpha_{n}+4 n \chi_{0} \tilde{\alpha}_{n}\right] & =2\left(V^{\prime} j_{n}+n U \tilde{j}_{n}\right)+e^{\alpha}\left(n^{2}-4\right) \dot{\nu}_{n} .
\end{aligned}
$$

In the case of the scalar modes the Lagrange multipliers are $k_{n}, g_{n}, \beta_{n}$ leaving five genuine variables. The transverse traceless gauge for the gravitational degrees of freedom dictates for the scalar modes $a_{n}=b_{n}=0$ and we will choose $\sigma_{n}=0$ for the YM modes. The remaining modes $\left(\gamma_{n}, \xi_{n}, \beta_{n}, g_{n}, k_{n}\right)$ fulfill :

$$
\begin{aligned}
& \frac{e^{\alpha}}{N_{0}} \frac{d}{d t}\left(\frac{e^{\alpha}}{N_{0}}\left(\dot{\gamma}_{n}+\frac{1}{3} \beta_{n}\right)\right)+\frac{2}{3}\left(n^{2}+5+9 U\right) \gamma_{n}+\frac{4}{3}\left(n^{2}-4\right) \xi_{n}=\frac{e^{2 \alpha}}{N_{0}^{2}} \dot{\chi}_{0} \dot{g}_{n}-2 V^{\prime} g_{n} \\
& -\frac{2 e^{\alpha}}{3 N_{0}^{2}} \dot{\chi}_{0} k_{n} \\
& \frac{e^{\alpha}}{N_{0}} \frac{d}{d t}\left(\frac{e^{\alpha}}{N_{0}}\left(\dot{\xi}_{n}-\frac{1}{6} \beta_{n}\right)\right)+\frac{1}{6}\left(n^{2}-1\right)\left(\gamma_{n}+2 \xi_{n}\right)=-\frac{e^{\alpha}}{6 N_{0}^{2}} \dot{\chi}_{0} k_{n} \\
& \beta_{n}=\frac{1}{n^{2}-1+2 U}\left[4\left(n^{2}-4\right) \dot{\xi}_{n}-\left(n^{2}-1\right) \dot{\gamma}_{n}+\left(n^{2}-1\right) \dot{\chi}_{0} g_{n}+e^{-\alpha} V^{\prime} k_{n}\right], \\
& k_{n}=\frac{1}{e^{2} \dot{\alpha} e^{\alpha}}\left[\frac{1}{2} \dot{\chi}_{0}\left(3 \dot{\gamma}_{n}+\beta_{n}\right)+\frac{3 N_{0}^{2}}{2 e^{2 \alpha}} V^{\prime} \gamma_{n}-\frac{3}{2} \dot{\chi}_{0}^{2} g_{n}\right]+3 \dot{\alpha} e^{\alpha} g_{n}, \\
& g_{n}=\frac{1}{e^{2} \dot{\alpha} e^{2 \alpha}}\left[\dot{\chi}_{0} \gamma_{n}+2 \frac{n^{2}-4}{n^{2}-1} \dot{\chi}_{0} \xi_{n}+\frac{1}{2} \frac{1}{n^{2}-1} V^{\prime} \beta_{n}-\frac{2}{e^{\alpha}} \frac{1}{n^{2}-1} V k_{n}\right]+\frac{1}{\dot{\alpha} e^{\alpha}} \frac{1}{n^{2}-1} k_{n} \text {. }
\end{aligned}
$$

To calculate the wave function we need the value of the Euclidean action for the classical solution which in our gauge is given by :

$$
\begin{aligned}
{ }^{T} S_{c l}^{(n)} & =\frac{V_{S^{3}}}{2} e^{2 \alpha}\left(d_{n} d_{n}^{\prime}+4 \alpha^{\prime} d_{n}^{2}\right)+\frac{V_{S^{3}}}{2 e^{2}}\left(\mu_{n} \mu_{n}^{\prime}-2 \chi_{0}^{\prime} d_{n} \mu_{n}\right), \\
{ } S_{c l}^{(n)} & =\frac{V_{S^{3}} e^{\alpha}}{2 e^{2} N_{0}}\left(n^{2}-4\right)\left(\dot{\nu}_{n}-\alpha_{n}\right) \nu_{n}, \\
{ }^{S} S_{c l}^{(n)} & =\frac{V_{S^{3}} e^{\alpha}}{e^{2} N_{0}}\left[\frac{1}{4}\left(\dot{\gamma}_{n}+\frac{1}{3} \beta_{n}-\dot{\chi}_{0} g_{n}\right) \gamma_{n}+2 \frac{n^{2}-4}{n^{2}-1}\left(\dot{\xi}_{n}-\frac{1}{6} \beta_{n}\right) \xi_{n}\right] .
\end{aligned}
$$


In the above expressions the prime denotes the derivative with respect to the conformal time $\eta$ with $\frac{d \eta}{d t}=-i \frac{N_{0}}{e^{\alpha}}$.

We proceed to solve the above system of equations in the large $n$ approximation, i. e. we only consider the terms with the highest occurring power of $n$. After eliminating the auxiliary modes the equations decouple in this approximation and can be written in terms of the gauge invariant variables introduced in eq. (67). They take the simple form $P_{n}^{\prime \prime}=n^{2} P_{n}$ for $P_{n}=d_{n}, \mu_{n}, R_{n}, \Gamma_{n}$. Imposing the Hartle-Hawking boundary condition $P_{n} \rightarrow 0$ for $\eta \rightarrow-\infty$ singles out the solutions $P_{n} \sim \exp (n \eta)$. With the eqs. (83) this results in the wave function

$$
\begin{aligned}
{ }^{T} \Psi^{(n)} & =C \exp \left[-\frac{V_{S^{3}}}{2} e^{2 \alpha} n d_{n}^{2}-\frac{V_{S^{3}}}{2 e^{2}} n \mu_{n}^{2}\right], \\
{ }^{V} \Psi^{(n)} & =C^{\prime} \exp \left[-\frac{V_{S^{3}}}{4 e^{2}} n R_{n}^{2}\right], \\
{ }^{S} \Psi^{(n)} & =C^{\prime \prime} \exp \left[-\frac{V_{S^{3}}}{6 e^{2}} n \Gamma_{n}^{2}\right] .
\end{aligned}
$$

We see that the perturbations start out in their ground state. In contrast to the gravitational mode $d_{n}$ the frequencies corresponding to the YM perturbations do not depend on the scale factor $e^{\alpha}$. This behavior is a consequence of conformal invariance of YM theory.

A case of particular interest is when the classical field $\chi_{0}$ obtains its minimum value $\chi_{0}= \pm 1$. Obviously this represents a solution of the background equation of motion (73) compatible with the Hartle-Hawking boundary condition. As already discussed, the Lagrangian in such a case can be explicitly expressed in terms of the gauge invariant variables. An additional simplification occurs because $\chi_{0}= \pm 1$ corresponds to a symmetric field $A^{(0)}$ which is pure gauge as can be seen from the eqs. (16), (17). This causes the decoupling of gravitational and gauge perturbations in the quadratic terms we are considering here. The Lagrangian $L_{Y M}^{n}={ }^{T} \mathcal{L}^{n}+{ }^{V} \mathcal{L}^{n}+{ }^{S} \mathcal{L}^{n}$ then takes the form :

$$
\begin{aligned}
{ }^{T} \mathcal{L}^{n}= & \frac{1}{2}\left[\frac{e^{\alpha}}{N_{0}} \dot{\mu}_{n}^{2}-\frac{N_{0}}{e^{\alpha}}\left(\left(n^{2}+1\right) \mu_{n}^{2}+2 n \chi_{0} \mu_{n} \tilde{\mu}_{n}\right)\right] \\
{ }^{V} \mathcal{L}^{n}= & \frac{e^{\alpha}}{2 N_{0}}\left[\dot{R}_{n}^{2}-4 \chi_{0} \dot{R}_{n} A_{n}-2 n \dot{R}_{n} \tilde{A}_{n}+2 n^{2} A_{n}^{2}+4 \chi_{0} n A_{n} \tilde{A}_{n}\right] \\
& -\frac{N_{0}}{4 e^{\alpha}}\left[n^{2} R_{n}^{2}-2 n \chi_{0} R_{n} \tilde{R}_{n}\right], \\
{ }^{S} \mathcal{L}^{n}= & \frac{e^{\alpha}}{2 N_{0}}\left[\frac{n^{2}-4}{n^{2}-1} \dot{\Gamma}_{n}^{2}+\frac{1}{3\left(n^{2}-1\right)} \dot{S}_{n}^{2}-\frac{2\left(n^{2}-4\right)}{3\left(n^{2}-1\right)} B_{n} \dot{\Gamma}_{n}-\frac{2}{3\left(n^{2}-1\right)} \chi_{0} B_{n} \dot{S}_{n}+\frac{1}{6} B_{n}^{2}\right] \\
& -\frac{N_{0}}{6 e^{\alpha}}\left[\left(n^{2}-4\right) \Gamma_{n}^{2}+S_{n}^{2}\right] .
\end{aligned}
$$


Starting from these expressions it is easy to calculate the equations of motion and eliminate the Lagrange multipliers $A_{n}$ and $B_{n}$. Solutions can be found without any further approximation and we obtain the explicitly gauge invariant wave function

$$
\begin{aligned}
{ }^{T} \Psi^{(n)} & \sim \exp \left[-\frac{V_{S^{3}}}{2 e^{2}}\left(n \mu_{n}^{2}+\chi_{0} \mu_{n} \tilde{\mu}_{n}\right)\right], \\
{ }^{V} \Psi^{(n)} & \sim \exp \left[-\frac{V_{S^{3}}}{4 e^{2}}\left(n R_{n}^{2}-2 \chi_{0} R_{n} \tilde{R}_{n}\right)\right], \\
{ }^{S} \Psi^{(n)} & \sim \exp \left[-\frac{V_{S^{3}}}{6 e^{2}}\left(\Gamma_{n}, S_{n}\right) M^{(n)}\left(\begin{array}{c}
\Gamma_{n} \\
S_{n}
\end{array}\right)\right],
\end{aligned}
$$

with

$$
M^{(n)}=\frac{1}{n\left(n^{2}-1\right)}\left(\begin{array}{cc}
\left(n^{2}+2\right)\left(n^{2}-4\right) & -\chi_{0}\left(n^{2}-4\right) \\
-\chi_{0}\left(n^{2}-4\right) & n^{2}-2
\end{array}\right) .
$$

As a consistency check one can apply the linear gauge constraints $\mathcal{H}_{\alpha}^{(n)}, \mathcal{H}_{\beta}^{(n)}$ and one finds that they annihilate ${ }^{V} \Psi^{(n)}$ and ${ }^{S} \Psi^{(n)}$.

Finally we want to discuss some features of the general solutions to the non-trivial systems of differential equations which govern the classical evolution of the perturbations. The solutions considered above have the drawback that they describe situations, where the gravitational and gauge sectors are completely decoupled as for the symmetric configuration. The separation was due to the fact that the YM Lagrangian is scale invariant and the only symmetric gravitational degree of freedom is that of the scale factor. In the first case the $n^{2}$-approximation is the first term of an approximation in rapidly changing fluctuations and the decoupling appears as a consequence of the diagonality of the kinetic terms [. The splitting in the case where $\chi_{0}$ is fixed to the minimum of the potential is again a property of the YM Lagrangian, together with the restriction to terms up to second order in the non-symmetric fields: From the general from of the YM action each term containing gravitational and gauge perturbations has to be supplemented by a component of the background field strength. It is therefore multiplied with a time derivative of $\chi_{0}$ or a term proportional to $\chi_{0}^{2}-1$ which both vanish for $\chi_{0}= \pm 1$. This effect is an intrinsic property of the symmetric YM potential and its knowledge in the formalism used in this paper is crucial as opposed to the approach, where the YM degrees of freedom are treated from the beginning as scalar fields. Obviously the above reasonings do not apply

\footnotetext{
${ }^{6}$ In fact this approximation reduces to a large momentum one for large enough $n$ due to local Lorentz invariance.
} 
to expressions of higher order in the perturbations and therefore there is no reason that the decoupling survives even in these special cases.

To investigate the interactions between the gauge and gravitational perturbations let us consider the next-to-leading-order equations in a large $n$ approximation of the coupled system describing the tensor modes, which are relevant for the discussion of density perturbations. Invoking the ansatz

$$
\begin{aligned}
d_{n}^{ \pm} & \equiv 1 / 2\left(d_{n}^{(\text {even })} \pm d_{n}^{(\text {odd })}\right), & \mu_{n}^{ \pm} & \equiv 1 / 2\left(\mu_{n}^{(\text {even })} \pm \mu_{n}^{(\text {odd })}\right) \\
& =e^{n \eta} D_{n}^{ \pm} & & =e^{n \eta} M_{n}^{ \pm},
\end{aligned}
$$

we find the following set of equations at order $n$ :

$$
\begin{aligned}
\frac{d}{d \eta} D_{n}^{ \pm}-\tanh \left(\eta-\eta_{a}\right) D_{n}^{ \pm}+\frac{H^{2}}{e^{2}} \cosh \left(\eta-\eta_{a}\right)^{2} f^{ \pm} M_{n}^{ \pm} & =0 \\
\frac{d}{d \eta} M_{n}^{ \pm}+g^{ \pm} M_{n}^{ \pm}+h^{ \pm} D_{n}^{ \pm} & =0
\end{aligned}
$$

where $d \eta=i N_{0} a^{-1} d t$. We recall at this point that the exponential $e^{n \eta}$ in equation (88) is the leading order behaviour found previously, while the prefactors $M_{n}^{ \pm}$and $D_{n}^{ \pm}$ represent the next-to-leading-order corrections. In eqs. (89) the conformal mode $a$ has been replaced by the classical solution

$$
a^{-1}(\eta)=H \cosh \left(\eta-\eta_{a}\right)
$$

where $\eta_{a}$ is defined by the value of $a$ on the given three-surface, $a(0)=a_{0}$. Inspection of equations (89), (90) reveals that the functions $D_{n}^{ \pm}$and $M_{n}^{ \pm}$do not really depend on $\mathrm{n}$; from now on we simply denote them by $D_{ \pm}, M_{ \pm}$. Inspection of the same equations shows that, unlike the leading order exponential, the prefactors depend on the value of the field $\chi_{0}(\eta)$. This field will be replaced by the classical solution given in ref. [9], expressed through the functions $f, g$ and $h$ defined below:

$$
f^{ \pm}= \pm\left(\chi_{0}^{2}(\eta)-1\right)+\chi_{0}^{\prime}(\eta), \quad-g^{ \pm}= \pm \chi_{0}(\eta), \quad h^{ \pm}= \pm\left(\chi_{0}^{2}(\eta)-1\right)-\chi_{0}^{\prime}(\eta)
$$

There are four different solutions, determined by their boundary values at $\eta=\infty$ and $\eta=0$ :

$$
\begin{aligned}
& I \quad: \quad \chi_{0}(\eta)=\operatorname{coth}\left(\eta-\eta_{0}\right), \quad \chi_{0}<-1, \\
& I I: \chi_{0}(\eta)=\tanh \left(\eta-\eta_{0}\right), \quad\left|\chi_{0}\right| \leq 1, \\
& \text { III : } \chi_{0}(\eta)=-\tanh \left(\eta-\eta_{0}\right), \quad\left|\chi_{0}\right| \leq 1, \\
& I V: \chi_{0}(\eta)=-\operatorname{coth}\left(\eta-\eta_{0}\right), \quad \chi_{0}>1 .
\end{aligned}
$$


On the other hand, $\eta_{0}$ expresses the boundary condition on $\chi_{0}(\eta)$ at $\eta=0: \chi_{0}(0)=\chi_{0}$. For instance, for the first of the above solutions, one gets: $\operatorname{coth}\left(-\eta_{0}\right)=\chi_{0}$; this defines $\eta_{0}$ for this solution. We should remark that $\chi_{0}(\eta)$ will remain in the region from which it started off at $\eta=0$. The solutions of eqs. (89) corresponding to the four possible choices for $\chi_{0}(\eta)$ are

$$
\begin{aligned}
& \underline{I}: \\
& D_{+}=A_{+} \cosh \left(\eta-\eta_{a}\right) \\
& M_{+}=A_{+}\left[\cosh \left(\eta_{0}-\eta_{a}\right) \sinh \left(\eta-\eta_{0}\right)^{-1}+2 \sinh \left(\eta_{0}-\eta_{a}\right) \cosh \left(\eta-\eta_{0}\right)\right] \\
& +B_{+} \sinh \left(\eta-\eta_{0}\right) \\
& D_{-}=-\frac{H^{2}}{e^{2}} A_{-}\left[\cosh \left(\eta_{0}-\eta_{a}\right) \cosh \left(\eta-\eta_{a}\right) \sinh \left(\eta-\eta_{0}\right)^{-2}\right. \\
& \left.+2 \sinh \left(\eta_{0}-\eta_{a}\right) \cosh \left(\eta-\eta_{a}\right) \operatorname{coth}\left(\eta-\eta_{0}\right)\right]+B_{-} \cosh \left(\eta-\eta_{a}\right), \\
& M_{-}=A_{-} \sinh \left(\eta-\eta_{0}\right)^{-1}, \\
& \text { II: } \\
& D_{+}=A_{+} \cosh \left(\eta-\eta_{a}\right), \\
& M_{+}=A_{+}\left[2 \cosh \left(\eta_{0}-\eta_{a}\right) \sinh \left(\eta-\eta_{0}\right)\right. \\
& \left.-\sinh \left(\eta_{0}-\eta_{a}\right) \cosh \left(\eta-\eta_{0}\right)^{-1}\right]+B_{+} \cosh \left(\eta-\eta_{0}\right), \\
& D_{-}=-\frac{H^{2}}{e^{2}} A_{-}\left[2 \cosh \left(\eta_{0}-\eta_{a}\right) \cosh \left(\eta-\eta_{a}\right) \tanh \left(\eta-\eta_{0}\right)\right. \\
& \left.-\sinh \left(\eta_{0}-\eta_{a}\right) \cosh \left(\eta-\eta_{a}\right) \cosh \left(\eta-\eta_{0}\right)^{-2}\right]+B_{-} \cosh \left(\eta-\eta_{a}\right), \\
& M_{-}=A_{-} \cosh \left(\eta-\eta_{0}\right)^{-1} \text {, }
\end{aligned}
$$

$\underline{I I I}:$

$$
\begin{aligned}
D_{+}= & \frac{H^{2}}{e^{2}} A_{+}\left[2 \cosh \left(\eta_{0}-\eta_{a}\right) \cosh \left(\eta-\eta_{a}\right) \tanh \left(\eta-\eta_{0}\right)\right. \\
& \left.-\sinh \left(\eta_{0}-\eta_{a}\right) \cosh \left(\eta-\eta_{a}\right) \cosh \left(\eta-\eta_{0}\right)^{-2}\right]+B_{+} \cosh \left(\eta-\eta_{a}\right), \\
M_{+}= & A_{+} \cosh \left(\eta-\eta_{0}\right)^{-1}, \\
D_{-}= & A_{-} \cosh \left(\eta-\eta_{a}\right) \\
M_{-}= & -A_{-}\left[2 \cosh \left(\eta_{0}-\eta_{a}\right) \sinh \left(\eta-\eta_{0}\right)\right. \\
& \left.-\sinh \left(\eta_{0}-\eta_{a}\right) \cosh \left(\eta-\eta_{0}\right)^{-1}\right]+B_{-} \cosh \left(\eta-\eta_{0}\right)
\end{aligned}
$$


$\underline{I V}:$

$$
\begin{aligned}
D_{+}= & \frac{H^{2}}{e^{2}} A_{+}\left[\cosh \left(\eta_{0}-\eta_{a}\right) \cosh \left(\eta-\eta_{a}\right) \sinh \left(\eta-\eta_{0}\right)^{-2}\right. \\
& \left.+2 \sinh \left(\eta_{0}-\eta_{a}\right) \cosh \left(\eta-\eta_{a}\right) \operatorname{coth}\left(\eta-\eta_{0}\right)\right]+B_{+} \cosh \left(\eta-\eta_{a}\right), \\
M_{+}= & A_{+} \sinh \left(\eta-\eta_{0}\right)^{-1}, \\
D_{-}= & A_{-} \cosh \left(\eta-\eta_{a}\right), \\
M_{-}= & -A_{-}\left[2 \sinh \left(\eta_{0}-\eta_{a}\right) \cosh \left(\eta-\eta_{0}\right)\right. \\
& \left.+\cosh \left(\eta_{0}-\eta_{a}\right) \sinh \left(\eta-\eta_{0}\right)^{-1}\right]+B_{-} \sinh \left(\eta-\eta_{0}\right),
\end{aligned}
$$

where $A_{ \pm}$and $B_{ \pm}$are integration constants. Note that there are two types of relations between coefficients entering both the gravitational and gauge perturbations: in the first case the relative strength depends on the ratio $H / e$ while it is universal in the second one. Insofar as the physical properties of these solutions are concerned, it is interesting to note that the interactions can cause quite strong correlations between the two kinds

of fluctuations. For instance, if the ratio $\frac{M_{ \pm}}{D_{ \pm}}$is normalized to a fixed value for a given conformal time $\eta$, it is predicted for any other value of $\eta$ and can differ substantially from the initial choice. This might give rise to interesting consistency conditions for cosmological models, if this ratio is constrained for physical reasons at two different times of the evolution.

Starting from the general expression (83) for the classical action and eliminating the derivatives via the eqs. $(89,90)$ one finds the tensor wave function in the $O(n)$ approximation :

$$
\begin{aligned}
{ }^{T} \Psi^{(n)}= & C \exp \left[-\frac{V_{S^{3}}}{2} e^{2 \alpha}\left(n+3 \alpha^{\prime}\right) d_{n}^{2}\right. \\
& \left.-\frac{V_{S^{3}}}{2 e^{2}}\left(n \mu_{n}^{2}+\chi_{0} \mu_{n} \tilde{\mu}_{n}+2\left(1-\chi_{0}^{2}\right) d_{n} \tilde{\mu}_{n}-2 \chi_{0}^{\prime} d_{n} \mu_{n}\right)\right] .
\end{aligned}
$$

As expected it shows a mixing between the gravitational and the YM mode in the new subleading terms.

\section{Conclusions and Outlook}

In this work we have extended the minisuperspace approximation treated in previous works to include the full infinite-dimensional model of symmetric gauge fields coupled to gravity. A primary ansatz characterizing our approach is that we treated gauge fields, such that the change of the gauge potential due to the translation on $S^{3}$ can be compensated 
by a suitable gauge transformation. $S O(N)$-symmetric gauge fields have been considered, however the extension to unitary, symplectic or exceptional groups is within reach; in fact it should be made, if a realistic model is to be constructed within this framework.

We restricted the gauge group to be just $S O(3)$, to keep the formulae tractable. We feel that this restriction does not hide too many characteristics of the system. Most probably, a general $S O(N)$ group would have richer topological structure, but we have not addressed this issue in this paper. Probably working with different class of gauge groups would give rise to essentially different phenomena. So, e. g., the groups $S U(N)$ with a conventional embedding of $S O(3)$ would impose the use of spinor harmonics.

Only a part of the superspace has been investigated, namely the portion where the minisuperspace variables are classical, while the perturbations are quantum mechanical. To investigate other regions, one should try to solve the Wheeler-DeWitt equation in a better approximation. This would allow taking into account the back reaction of the perturbations on the background fields. An interesting feature of our calculation has been the existence of gauge invariant variables and the possibility to express the second order Lagrangian in terms of them, illustrated in the main text for the case $\chi_{0}= \pm 1$.

We will present the detailed predictions of the wave function found above in a future publication. For the time being we comment on some very basic characteristics of this solution. The wave function has been found in the regime of large $\mathrm{n}$ (the order of the corresponding harmonic).

As a first step, we only kept the terms of order $n^{2}$; the wave function we found shows that the perturbations start off in their ground state. This means that approximating the inhomogeneous and anisotropic degrees of freedom just to second order is well justified and describes the system quite well in the relevant region of the superspace. We also point out that the vector perturbations are genuine in our case and not a gauge artifact, as is the case of the scalar fields.

When we proceed with the next-to-leading order, i. e. we also keep terms of order $n$, we encounter new, quite interesting, phenomena. Let us pay particular attention to the tensor perturbation modes, which are e. g. relevant for the spectrum of relic gravitons in the universe. We stress that, in contrast to models with scalar fields, we have a second tensor mode in the theory stemming from the YM field. In the next to leading order this mode couples to the gravity tensor perturbation often referred to as the linear graviton. A similiar behaviour shows up in the corresponding tensor mode wave function. It is conceivable that these effects could have interesting cosmological consequences.

One line of development of our work would be the inclusion of scalar and fermionic fields and consider the full system at work. 
Moreover, one may calculate wormhole effects using our results. For example one can find the effective interactions between gauge fields at large distances caused by the presence of wormholes. This requires calculating the matrix element of the product of two gauge field operators between the flat space vacuum state and wormhole states. The latter one can be computed semiclassically starting from our equations of motion for the perturbations and finding solutions which fulfill the boundary conditions appropriate for wormhole states.

Acknowledgement This work was partially supported by the Deutsche Forschungsgemeinschaft and the EC under contract no. SC1-CT92-0789 and the CEC Science Program no. SC1-CT91-0729.

\section{Appendices}

\section{A Symmetric Fields}

Let us briefly summarize here how one can perform the harmonic analysis of symmetric fields on homogeneous spaces. For the general method of constructing symmetric fields we refer the reader to refs. [11] where a detailed treatment is given. Let us just comment, that a field is called $S$ symmetric on a space $M \times S / R$ if a symmetry transformation by an element of the isometry group $S$ corresponds to a gauge transformation. This implies that functions of the fields such as the energy-momentum tensor or the Lagrangian density are independent of the coordinates of $S / R$ just because they are gauge invariant. The requirement that transformations of the fields under the action of the symmetry group of $S / R$ are compensated by gauge transformations, leads to certain constraints on the fields. The solution of these constraints provides the theory on $M$. In particular, a gauge field $A_{M}$ on $M^{D}$ splits into $A_{m}$ on $M$ and $A_{\alpha}$ on $S / R ; A_{m}$ behaves as a scalar under $S$ transformations and lies in the adjoint representation of the gauge group $G$. The surviving gauge symmetry $H$ on $M$ is that subgroup of $G$ which commutes with $R$. In other words the gauge group $H$ in one dimension is the centralizer of $R$ in $G$, i.e. $H=C_{G}\left(R_{G}\right) ; R_{G}$ is the isomorphic image of $R$ in $G$. The remaining components of the gauge field $A_{\alpha}$ become vectors under the coset space transformations. The transformation properties of the fields $A_{\alpha}$ under $H$ can be found if we express the adjoint irreducible representation of 
$G$ in terms of $R_{G} \times H$

$$
\begin{aligned}
G & \supset R_{G} \times H, \\
\operatorname{adj} G & =(\operatorname{adj} R, 1)+(1, \operatorname{adj} H)+\sum_{i}\left(R_{i}, H_{i}\right) .
\end{aligned}
$$

and $S$ under $R$

$$
\begin{array}{ll}
S & \supset R, \\
\operatorname{adj} S=\operatorname{adj} R+\sum_{i} S_{i} .
\end{array}
$$

Then for every pair $R_{i}, S_{i}$ where $R_{i}$ and $S_{i}$ are identical irreducible representations of $R$, there remains a multiplet on $M$ transforming under the representation $H_{i}$ of $H$. All other scalar fields vanish.

In order to perform the harmonic expansion of the fields on $M \times S / R$ we have to find a suitable ground state configuration around which to expand. Since the symmetry of the vacuum $M \times S / R$ is the symmetry group of $M$ times $S$, we demand that the background configuration $A_{M}^{B}$ should possess these symmetries. Therefore $A_{m}^{B}$ has to vanish while $A_{\alpha}^{B}$ has to be $S$-symmetric. Such a configuration is given by

$$
A_{\alpha}^{B}=e_{\alpha}^{i} J_{i}
$$

where $\alpha=1, \ldots, \operatorname{dim} S-\operatorname{dim} R, i=1, \ldots, \operatorname{dim} R, e_{\alpha}^{i}$ are vielbeins of the coset space and $J_{i}$ are the generators of the gauge group $G$ spanning the algebra of $R_{G}$. The background field $A_{\alpha}^{B}$ is also $S$-symmetric in the broader sense i.e. including a suitable gauge transformation. Indeed we obtain that

$$
\delta_{A} A_{\alpha}^{B}=-\left(\partial_{\alpha} \Omega_{A}^{i}\right) J_{i}+\left[\Omega_{A}^{i} J_{i}, A_{\alpha}^{B}\right],
$$

where $\Omega_{A}^{i}$ are the so called $R$-compensators, and therefore the gauge transformation needed to compensate this $S$-transformation is given by :

$$
W_{A}=-\Omega_{A}^{i} J_{i}
$$

Let us now discuss the harmonic expansion of a field on a coset space. It is well known that the matrix elements $D_{p q}^{(m)}$ of the inequivalent unitary irreducible representations of $S$, indexed by $(m)$, serve as a complete orthonormal basis into which any function defined on $S$ can be expanded (this is known as the Peter-Weyl theorem in group theory [16]). Since $S$ is the isometry group of $S / R$, we can still use, in the case of the coset space, the matrix elements of the unitary irreducible representations of $S$ as an orthonormal basis for the harmonic analysis, although these now have to be properly restricted [17]. The 
harmonic expansions of the fields $A_{m}, A_{a}$ can be written as

$$
\begin{aligned}
& A_{m}(x, y)=\sum_{m^{\prime}} \sum_{p q} \sqrt{\frac{d_{m^{\prime}}}{d_{\mathcal{D}}}} D_{p q}^{\left(m^{\prime}\right)}(L(y)) a_{m}{ }_{p q}^{\left(m^{\prime}\right)}(x) \\
& A_{a}(x, y)=A_{a}^{B}+\sum_{m} \sum_{p q} \sqrt{\frac{d_{m}}{d_{\mathcal{D}}}} D_{p q}^{(m)}(L(y)) \phi_{a}{ }_{p q}^{(m)}(x)=A_{a}^{B}+\bar{A}_{a},
\end{aligned}
$$

where $L(y)$ is a representative element of each $R$-equivalence class, $d_{m}$ is the dimension of the representation $m$ of $S$ and $d_{\mathcal{D}}$ is the dimension of the $\mathcal{D}$ representation of $R$. As we will see, only those irreducible representations of $S$ contribute to the harmonic expansion of a field on $S / R$ in the representation $\mathcal{D}$ of $R$, which contain $\mathcal{D}$ in their decomposition under $R$. Furthermore, all the relevant $D^{(m)}$ 's contribute to the harmonic expansion only with those of their rows which correspond to the particular $R$-representation $\mathcal{D}$. In order to determine the relevant representations of $S$ which contribute to the harmonic expansions (A.6), (A.7), we should demand that under a general $S$-transformation, which now includes the gauge transformation given in eq. (A.5), the coefficients in these expansions should transform according to some irreducible representation of $S$ [18]. We find :

$$
\begin{aligned}
D_{p s}^{(m)}\left(Q_{i}\right) \phi_{a} \underset{p q}{(m)}+f_{i a b} \phi_{b} \underset{s q}{(m)}-\left[J_{i}, \phi_{a} \underset{s q}{(m)}\right]=0 \\
D_{p s}^{(m)}\left(Q_{i}\right) a_{m} \underset{p q}{(m)}-\left[J_{i}, a_{m} \underset{s q}{(m)}\right]=0 .
\end{aligned}
$$

Eqs. (A.8), (A.9) specify which representations of $S$ are present in the harmonic expansions of the corresponding fields given in eqs. (A.6), A.7). From the solution of eq. (A.8) we find that the relevant representations of $S$ for the harmonic expansion of $A_{a}$ are those which include in their restriction to $R$ the representations of $R$ appearing in the cross product of $R_{i}$ and $S_{i}$ given in eqs. (A.1) and (A.2). Similarly, for the harmonic expansion of $A_{m}$ one should include only those representations of $R$ which appear in eq. (A.1). All these $S$ representations will contribute only with their rows which correspond to the $R$

representation. Furthermore, the $S$-symmetric fields are the $y$-independent ones, i.e. the first terms in the expansions (A.6), (A.7).

\section{B Harmonics}

Various versions of the harmonics on $S^{3}$ have appeared in the literature [19, [13, 20]. We use the ones basically proposed in [13], which bear great similarity to the harmonics used by Halliwell and Hawking, but their properties are much more transparent and easy to derive from group theoretical considerations. We refer the reader to [13] for a more complete account and present here some basic steps, needed to adapt these considerations 
to our notation. The manifold is $S^{3}$, or $S U(2)$, which is considered as a coset space of $S U(2)_{L} \otimes S U(2)_{R}$ over $S U(2)_{\text {diag }}$, where $S U(2)_{\text {diag }}$ is defined as usual as the subgroup of $S U(2)_{L} \otimes S U(2)_{R}$ with equal left and right rotations. The harmonics for a field on the above coset corresponding to an angular momentum $J$, say, will transform as the irreducible representations $\mathcal{D}^{(J)}$ of $S U(2)_{\text {diag }}$. Such representations may be sieved out of the tensor product representations $D^{(m)}$ of the group $S U(2)_{L} \otimes S U(2)_{R}$ which contain $\mathcal{D}^{(J)}$ upon restriction to $S U(2)_{\text {diag }}$. The tensor representations are characterized by the two angular momenta $j_{L}, j_{R}$ corresponding to $S U(2)_{L}, S U(2)_{R}$ respectively. For instance the scalar harmonics (corresponding to $J=0$ ) are contained in

$$
(0,0),\left(\frac{1}{2}, \frac{1}{2}\right),(1,1),\left(\frac{3}{2}, \frac{3}{2}\right), \ldots,
$$

while the vector harmonics $(J=1)$ are contained in

$$
(0,1),(1,0),(1,1),\left(\frac{1}{2}, \frac{1}{2}\right),\left(\frac{1}{2}, \frac{3}{2}\right), \ldots,
$$

As a matter of notation, we denote the harmonic characterized by $J$ constructed out of the pair $\left(j_{L}, j_{R}\right)$, by $D_{p q}^{\left(j_{L}, j_{R} \mid J \xi\right)}(L(y))$. The indices $p$ and $q$ label matrix elements and $L(y)$ is the representative element for the coset, at the point described by $y$ in some local coordinate system. The additional index $\xi$ is used to distinguish between the various $\mathcal{D}^{(J)}$ 's that may be contained in $D^{(m)}$. In fact for the $S U(2)$ group only one $\mathcal{D}^{(J)}$ appears for fixed $J$, so we completely drop this index in the sequel. We also note that the index $p$ is constrained to run only over the $2 J+1$ values corresponding to the relevant $\mathcal{D}^{(J)}$. As explained in Appendix A, any field on the coset space transforming according to $\mathcal{D}^{(J)}$ can be expanded as follows :

$$
\psi_{p}^{(J)}(L(y))=\sum_{\left(j_{L}, j_{R}\right) \supset J} \sum_{q} \psi_{q}^{\left(j_{L}, j_{R} \mid J\right)} D_{p q}^{\left(j_{L}, j_{R} \mid J\right)}(L(y)) .
$$

The orthonormality relation reads :

$$
\sum_{p} \int_{S^{3}} d \mu(y)\left[D_{p q}^{\left(j_{L}, j_{R} \mid J\right)}(L(y))\right]^{*}\left[D_{p q^{\prime}}^{\left(j_{L}^{\prime}, j_{R}^{\prime} \mid J\right)}(L(y))\right]=\frac{V_{S^{3}}(2 J+1)}{\left(2 j_{L}+1\right)\left(2 j_{R}+1\right)} \delta^{j_{L} j_{L}^{\prime}} \delta^{j_{R} j_{R}^{\prime}} \delta_{q q^{\prime}} .
$$

As the reader will notice, we define in the sequel the transverse harmonics with a normalization equal to $\sqrt{\frac{\left(2 j_{L}+1\right)\left(2 j_{R}+1\right)}{(2 J+1)}}$. The motivation was simply to make the right hand side of eq. (B.4) equal $V_{S^{3}} \delta^{j_{L} j_{L}^{\prime}} \delta^{j_{R} j_{R}^{\prime}} \delta_{q q^{\prime}}$. It is useful to define the quantities

$$
n=j_{L}+j_{R}+1, \quad d=j_{L}-j_{R},
$$


in terms of which the Casimir operator of the $\left(j_{L}, j_{R}\right)$ representation of $S O(4) \approx S U(2)_{L} \otimes$ $S U(2)_{R}$ equals

$$
C_{2}\left(j_{L}, j_{R}\right)=n^{2}+d^{2}-1
$$

Two important results read :

$$
\begin{aligned}
\nabla_{a} D_{p q}^{\left(j_{L}, j_{R} \mid J\right)}(L(y)) & =-\left[T_{a}^{\left(j_{L}, j_{R}\right)}\right]_{p r} D_{r q}^{\left(j_{L}, j_{R} \mid J\right)}(L(y)) \\
\nabla_{a} \nabla^{a} D_{p q}^{\left(j_{L}, j_{R} \mid J\right)}(L(y)) & =\left[C_{2}\left(\mathcal{D}^{(J)}\right)-C_{2}\left(j_{L}, j_{R}\right)\right] D_{p q}^{\left(j_{L}, j_{R} \mid J\right)}(L(y)) \\
& =\left[J(J+1)-\left(n^{2}+d^{2}-1\right)\right] D_{p q}^{\left(j_{L}, j_{R} \mid J\right)}(L(y)) .
\end{aligned}
$$

In the above the $T_{a}^{\left(j_{L}, j_{R}\right)}$ are the part of the reductive decomposition $\left(T_{a}^{\left(j_{L}, j_{R}\right)}, T_{i}^{\left(j_{L}, j_{R}\right)}\right)$ of the generators of $\left(j_{L}, j_{R}\right)$, which corresponds to $S U(2)_{\text {diag }} ; C_{2}\left(\mathcal{D}^{(J)}\right)=J(J+1)$ is the Casimir operator for the representation $J$ defining the harmonic. These important results mean that any relation involving differentials on $S^{3}$ reduces to mere algebra. Two more identities are needed to find the properties of the harmonics. These are the commutation relations for covariant derivatives acting on vectors and tensors :

$$
\begin{gathered}
\left(\nabla_{a} \nabla_{c}-\nabla_{c} \nabla_{a}\right) V_{b}=V_{d} R_{b a c}^{d} \\
\left(\nabla_{a} \nabla_{b}-\nabla_{b} \nabla_{a}\right) H_{c d}=H_{e d} R_{c a b}^{e}+H_{c e} R_{d a b}^{e} \\
R_{a b c d}=\delta_{b c} \delta_{a d}-\delta_{a c} \delta_{b d} .
\end{gathered}
$$

In the following we give explicitly some properties of the scalar, vector and tensor harmonics, which are the only relevant ones for the case $G=S O(N)$, whose special case $N=3$ is considered in this work.

\section{1) Scalar harmonics}

These are scalar eigenfunctions of the Laplacian. It is convenient to define them with the following normalization :

$$
Q_{q}^{(n)}(L(y))=n^{2} D_{0 q}^{\left(j_{L}, j_{R} \mid 0\right)}(L(y))
$$

The first index on $D_{0 q}^{\left(j_{L}, j_{R} \mid 0\right)}(L(y))$ takes the value corresponding to the identity representation, so there is no point in keeping it in the notation for the harmonic $Q_{q}^{(n)}$. We also use the index $(n)$ defined above to characterize the scalar harmonics, rather than the pair $\left(j_{L}, j_{R}\right)$. In fact for the scalar harmonics, $j_{L}=j_{R} \equiv j$, so the other combination, namely $d$, vanishes in this case. The quantity $j$ runs over integer and half-odd-integer values. For the normalization introduced above, it is easy to show that

$$
\int_{S^{3}} d \mu(y)\left[Q_{q}^{(n)}(L(y))\right]^{*}\left[Q_{q^{\prime}}^{\left(n^{\prime}\right)}(L(y))\right]=V_{S^{3}} \delta^{n n^{\prime}} \delta_{q q^{\prime}}
$$


The eigenvalue of the Laplacian is found from the difference of the Casimir operators (eq. (B.7)) :

$$
\nabla_{a} \nabla^{a} Q_{q}^{(n)}(L(y))=-\left(n^{2}-1\right) Q_{q}^{(n)}(L(y))
$$

\section{2) Vector harmonics}

The vector harmonics on a simply connected coset space without boundary can be uniquely decomposed into a sum of the "transverse vector harmonics", characterized by the vanishing of their covariant divergence and the "longitudinal vector harmonics", given by the gradient of some (scalar) function. This corresponds to the fact, obvious from eq. (B.2), that the quantity $d$ may take three values, namely $-1,0$, and +1 . Thus the vector harmonics split into categories, according to the value of $d$. The ones corresponding to $d=-1(d=+1)$ are called odd (even) transverse vector harmonics; we denote them by $S_{a \mid q}^{(n-)}(L(y)), S_{a \mid q}^{(n+)}(L(y))$ respectively, while for $d=0$ we get the longitudinal vector harmonics, $P_{a \mid q}^{(n)}(L(y))$. The index a runs from 1 to 3 . We note that the terms "odd" ("even") derive from the behavior of the corresponding harmonics under a parity transformation [19]. Let us now be more specific and give the exact definitions of the various harmonics :

$$
S_{a \mid q}^{(n \pm)}(L(y))=\sqrt{\frac{n^{2}-1}{3}} D_{a q}^{(j \pm 1, j \mid 1)}(L(y)) .
$$

On the other hand, since the scalar harmonics, previously defined, span the space of scalar functions on the coset space, the longitudinal vector harmonic may be chosen to be the gradient of a scalar harmonic :

$$
P_{a \mid q}^{(n)}(L(y))=\frac{1}{n^{2}-1} \nabla_{a} Q_{q}^{(n)}(L(y)) .
$$

Using the above definitions, we obtain the following properties of the vector harmonics :

$$
\begin{aligned}
& \sum_{a} \int_{S^{3}} d \mu(y)\left[S_{a \mid q}^{(n \pm)}(L(y))\right]^{*}\left[S_{a \mid q^{\prime}}^{\left(n^{\prime} \pm\right)}(L(y))\right]=V_{S^{3}} \delta^{n n^{\prime}} \delta_{q q^{\prime}}, \\
& \sum_{a} \int_{S^{3}} d \mu(y)\left[S_{a \mid q}^{(n \pm)}(L(y))\right]^{*}\left[S_{a \mid q^{\prime}}^{\left(n^{\prime} \mp\right)}(L(y))\right]=0 \\
& \sum_{a} \int_{S^{3}} d \mu(y)\left[P_{a \mid q}^{(n)}(L(y))\right]^{*}\left[P_{a \mid q^{\prime}}^{\left(n^{\prime}\right)}(L(y))\right]=\frac{V_{S^{3}}}{n^{2}-1} \delta^{n n^{\prime}} \delta_{q q^{\prime}}, \\
& \nabla^{a} S_{a \mid q}^{(n \pm)}(L(y)) \quad=\quad 0 \\
& \nabla^{a} P_{a \mid q}^{(n)}(L(y)) \quad=\quad-Q_{q}^{(n)}(L(y)), \\
& \varepsilon_{a c b} \nabla_{a} S_{b \mid q}^{(n \pm)}(L(y))=n S_{c \mid q}^{(n \mp)}(L(y))
\end{aligned}
$$




$$
\begin{aligned}
& \nabla_{a} \nabla^{a} S_{b \mid q}^{(n \pm)}(L(y))=-\left(n^{2}-2\right) S_{b \mid q}^{(n \pm)}(L(y)) \\
& \nabla_{a} \nabla^{a} P_{b \mid q}^{(n)}(L(y))=-\left(n^{2}-3\right) P_{b \mid q}^{(n)}(L(y))
\end{aligned}
$$

\section{3) Tensor harmonics}

As in the previous case, also the tensor harmonics can be classified into several kinds, according to the value of $d \equiv j_{L}-j_{R}$. This quantity may now take the values $\pm 2, \pm 1,0$.

The harmonics with $d=-2(d=+2)$ are called odd (even) transverse tensor harmonics. To get them from the $D_{p q}^{\left(j_{L}, j_{R} \mid 2\right)}$, we represent the index $p$ (which may take 5 values) by a pair of indices $a_{1} a_{2}$, each running from 1 to 3 . Then the definition is

$$
G_{a_{1} a_{2} \mid q}^{(n \pm)}(L(y)) \equiv \sqrt{\frac{n^{2}-4}{5}} \sum_{q_{1}, q_{2}} P\left(a_{1} a_{2} \mid q_{1} q_{2}\right) D_{\left(q_{1} q_{2}\right) q}^{(j \pm 2, j \mid 2)}(L(y))
$$

where the definition of the projection operator $P\left(a_{1} a_{2} \mid q_{1} q_{2}\right)$ reads :

$$
P\left(a_{1} a_{2} \mid q_{1} q_{2}\right) \equiv\left[\frac{1}{2}\left(\delta_{q_{1} a_{1}} \delta_{q_{2} a_{2}}+\delta_{q_{2} a_{1}} \delta_{q_{1} a_{2}}\right)-\frac{1}{3} \delta_{q_{1} q_{2}} \delta_{a_{1} a_{2}}\right]
$$

This operator projects out the symmetric and traceless part of the expression it acts on.

Thus the harmonics $G_{a_{1} a_{2} \mid q}^{(n \pm)}(L(y))$ are symmetric, traceless and transverse. We find the following additional properties of the transverse tensor harmonics :

$$
\begin{aligned}
\sum_{a_{1}, a_{2}} \int d \mu(y)\left[G_{a_{1} a_{2} \mid q}^{(n \pm)}(L(y))\right]^{*}\left[G_{a_{1} a_{2} \mid q^{\prime}}^{\left(n^{\prime}\right.}(L(y))\right] & =V_{S^{3}} \delta^{n n^{\prime}} \delta_{q q^{\prime}} \\
\sum_{a_{1}, a_{2}} \int d \mu(y)\left[G_{a_{1} a_{2} \mid q}^{(n \pm)}(L(y))\right]^{*}\left[G_{a_{1} a_{2} \mid q^{\prime}}^{\left(n^{\prime} \mp\right)}(L(y))\right] & =0 \\
\nabla_{a} \nabla^{a} G_{a_{1} a_{2} \mid q}^{(n \pm)}(L(y)) & =-\left(n^{2}-3\right) G_{a_{1} a_{2} \mid q}^{(n \pm)}(L(y)) \\
\nabla^{a_{1}} G_{a_{1} a_{2} \mid q}^{(n \pm)}(L(y)) & =0, \\
\delta_{a_{1} a_{2}} G_{a_{1} a_{2} \mid q}^{(n \pm)}(L(y)) & =n G_{a_{4} a_{2} \mid q}^{(n \mp)}(L(y)) .
\end{aligned}
$$

The remaining types of tensor harmonics can be expressed in the form :

$$
\sum_{q_{1}, q_{2}} P\left(a_{1} a_{2} \mid q_{1} q_{2}\right) \nabla_{q_{1}} D_{q_{2} \mid q}^{\left(j_{L}, j_{R} \mid 1\right)}(L(y))
$$

Basically they are covariant derivatives of harmonics with smaller "magnetic quantum numbers" $d$, properly symmetrized. We recall that any vector harmonic is a linear combination of the transverse and longitudinal vector harmonics. Thus these remaining harmonics are linear combinations of : 
1) The even and odd "longitudinal-transverse" harmonics, with $|d| \equiv\left|j_{L}-j_{R}\right|=1$. Eq. (B.22) becomes :

$$
S_{a_{1} a_{2} \mid q}^{(n \pm)}(L(y))=\frac{1}{2}\left[\nabla_{a_{1}} S_{a_{2}}^{(n \pm)}(L(y))+\nabla_{a_{2}} S_{a_{1}}^{(n \pm)}(L(y))\right]
$$

and

2) the "longitudinal-longitudinal" tensor harmonics, with $d \equiv j_{l}-j_{R}=0$. Now eq. (B.22) becomes

$$
P_{a_{1} a_{2} \mid q}^{(n)}(L(y))=\frac{1}{2}\left[\nabla_{a_{1}} P_{a_{2} \mid q}^{(n)}(L(y))+\nabla_{a_{2}} P_{a_{1} \mid q}^{(n)}(L(y))\right]-\frac{1}{3} \delta_{a_{1} a_{2}} \nabla^{a} P_{a \mid q}^{(n)}(L(y)),
$$

respectively. From the definition of $P_{a \mid q}^{(n)}(L(y))$ one can prove that

$$
P_{a_{1} a_{2} \mid q}^{(n)}(L(y))=\frac{1}{n^{2}-1} \nabla_{a_{1}} \nabla_{a_{2}} Q_{q}^{(n)}(L(y))+\frac{1}{3} \delta_{a_{1} a_{2}} Q_{q}^{(n)}(L(y)),
$$

which is the definition of this quantity used by Halliwell and Hawking.

In the following we gather some properties of these harmonics.

$$
\begin{aligned}
\sum_{a_{1}, a_{2}} \int d \mu(y)\left[S_{a_{1} a_{2} \mid q}^{(n \pm)}(L(y))\right]^{*}\left[S_{a_{1} a_{2} \mid q^{\prime}}^{\left(n^{\prime} \pm\right.}(L(y))\right] & =V_{S^{3}} \frac{\left(n^{2}-4\right)}{2} \delta^{n n^{\prime}} \delta_{q q^{\prime}}, \\
\sum_{a_{1}, a_{2}} \int d \mu(y)\left[S_{a_{1} a_{2} \mid q}^{(n)}(L(y))\right]^{*}\left[S_{a_{1} a_{2} \mid q^{\prime}}^{\left(n^{\prime}\right)}(L(y))\right]=0 & =0 \\
\sum_{a_{1}, a_{2}} \int d \mu(y)\left[P_{a_{1} a_{2} \mid q}^{(n)}(L(y))\right]^{*}\left[P_{a_{1} a_{2} \mid q^{\prime}}^{\left(n^{\prime}\right)}(L(y))\right]= & V_{S^{3}} \frac{2\left(n^{2}-4\right)}{3\left(n^{2}-1\right)} \delta^{n n^{\prime}} \delta_{q q^{\prime}}, \\
\nabla^{a_{1}} S_{a_{1} a_{2} \mid q}^{(n \pm)}(L(y)) & =-\left(n^{2}-4\right) S_{a_{2} \mid q}^{(n \pm)}(L(y)), \\
\nabla^{a_{1}} \nabla^{a_{2}} S_{a_{1} a_{2} \mid q}^{(n \pm)}(L(y)) & =0 \\
\nabla^{a} \nabla_{a} S_{a_{1} a_{2}}^{(n \pm)}(L(y)) & =-\left(n^{2}-6\right) S_{a_{1} a_{2} \mid q}^{(n \pm)}(L(y)), \\
\nabla^{a} \nabla_{a} P_{a_{1} a_{2} \mid q}^{(n)}(L(y)) & =-\left(n^{2}-7\right) P_{a_{1} a_{2} \mid q}^{(n)}(L(y)), \\
\delta_{a_{1} a_{2}} P_{a_{1} a_{2} \mid q}^{(n)}(L(y)) & =0, \\
\nabla^{a_{1}} P_{a_{1} a_{2} \mid q}^{(n)}(L(y)) & =-\frac{2}{3}\left(n^{2}-4\right) P_{a_{2} \mid q}^{(n)}(L(y)), \\
\nabla^{a_{1}} \nabla^{a_{2}} P_{a_{1} a_{2} \mid q}^{(n)}(L(y)) & =\frac{2}{3}\left(n^{2}-4\right) Q_{q}^{(n)}(L(y)) .
\end{aligned}
$$

\section{References}

[1] R. Arnowit, S. Deser and C. W. Misner, in Gravitation, ed. L. Witten (Wiley, New York, 1962). 
[2] J. B. Hartle and S. W. Hawking, Phys. Rev. D28 (1983) 2960.

[3] J.J. Halliwell and S. W. Hawking, Phys. Rev. D31 (1983) 1777.

[4] A. Vilenkin, Phys. Lett. B117 (1982) 25; Phys. Rev. D27 (1983) 2848; D30 (1984) 509; D33 (1986) 3560;D37 (1988) 888; Nucl. Phys. B252 (1985) 141; A. D. Linde, Zh. Eksp. Teor. Fiz. 87 (1984) 369 (Sov. Phys. JETP 60 (1984) 211); Nuov. Cim. 39 (1984) 401; Rep. Prog. Phys. 47 (1984) 925.

[5] See for example J.J. Halliwell, "Introductory Lectures on Quantum Cosmology" in Quantum Cosmology and Baby Universes, Jerusalem Winter School on Theor. Physics, S. Coleman, J.B. Hartle, T. Piran and S. Weinberg eds. and references therein.

[6] P.D. D'Eath and J.J. Halliwell, Phys. Rev. D35 (1987) 1100.

[7] J. Louko, Phys. Rev. D38 (1988) 478.

[8] O. Bertolami, J.M. Mourão, R.F. Picken and I.P. Volobujev, Int. J. Mod. Phys. A6, (1991) 4149.

[9] O. Bertolami and J.M. Mourão, Class. Quantum Grav. 8, (1991) 1271.

[10] M. Henneaux, J. Math. Phys. 23 (1982) 830.

[11] R. Coquereaux and A. Jadczyk, Riemannian Geometry, Fiber Bundles, Kaluza-Klein Theories and all that..., (World Scientific, Singapore, 1988); Y.A. Kubyshin, J.M. Mourão, G. Rudolph and I.P. Volobujev, Dimensional Reduction of Gauge Theories, Spontaneous Compactification and Model Building, (Springer Notes in Physics Vol. 349, Heidelberg 1989); D. Kapetanakis and G. Zoupanos, Phys. Rep. 219 (1992) 1.

[12] H. F. Dowker, Fermilab-preprint, FERMILAB-Pub-92/294-A, October 1992.

[13] R. Camporesi, Phys. Rep. 196 (1990) 1.

[14] J. Bardeen, Phys. Rev. D22 (1980) 1882;

For a review see : V. F. Mukhanov, H. A. Feldman and R. H. Brandenberger Phys. Rep. 215 (1991) 203.

[15] M. Lüscher and G. Münster Nucl. Phys. B232 (1984) 445. 
[16] N.J. Vilenkin, Special functions and the Theory of Group Representations, Translations of Mathematical Monographs, Vol. 22 (American Mathematical Society, Providence, Rhode Island, 1968).

[17] A. Salam and J. Strathdee, Ann. Phys. 141 (1982) 316.

[18] L. Palla, Z. Phys. C24 (1984) 195.

[19] E. M. Lifschitz and I. M. Khalatnikov, Adv. Phys. 12 (1963) 185; U. H. Gerlach and U. K. Sengupta, Phys. Rev. D18 (1978) 1773.

[20] J. S. Dowker and D. Pettengill, University of Manchester report, unpublished (1974); H. F. Dowker and R. Laflamme, Nucl. Phys. B366 (1991) 209; M. A. Rubin and C. R. Ordóñez, J. Math. Phys. 26 (1985) 65. 